\title{
ON SPECTRAL THEORY OF LAX OPERATORS ON SYMMETRIC SPACES: VANISHING VERSUS CONSTANT BOUNDARY CONDITIONS
}

\author{
VLADIMIR S. GERDJIKOV
}

Communicated by Metin Gürses

\begin{abstract}
We outline several specific issues concerning the theory of multicomponent nonlinear Schrödinger equations with vanishing and constant boundary conditions. We start with the spectral properties of the Lax operator $L$ for vanishing boundary conditions. We introduce the fundamental analytic solutions (FAS) and demonstrate their importance for relating the scattering problem to a Riemann-Hilbert problem, and for the construction of the resolvent of $L$. Then we generalize this procedure to constant boundary conditions case. We start with the structure of the class of allowed potentials $\mathcal{M}$ and give a recipe of how FAS can be constructed on each of the leafs of the relevant Riemannian surface. This allows us to relate the scattering problem to a Riemann-Hilbert problem posed on a Riemannian surface. Next we use these FAS to construct the resolvent of $L$ and study its spectral properties. We also introduce the minimal set of scattering data on the continuous spectrum of $L$ which generically has varying multiplicity. The general construction is illustrated by three representative examples related to A.III, C.II and D.III symmetric spaces. Finally we consider regularized Wronskian relations which allow us to analyze the mapping between the potential of $L$ and the scattering data.
\end{abstract}

\section{Contents}

1 Introduction $\quad 2$

2 Algebraic Preliminaries 5

3 Spectral Properties of Lax Operators on Symmetric Spaces. $\begin{array}{ll}\text { Vanishing Boundary Conditions } & 8\end{array}$

3.1 Jost Solutions, Scattering Matrix and FAS . . . . . . . . . . . 8

3.2 The Fundamental Analytic Solutions . . . . . . . . . . . . . 10 
3.3 The FAS and Higher Representations . . . . . . . . . . . . . . . . 14

3.4 Typical Reductions . . . . . . . . . . . . . . . . 15

3.5 The Resolvent of $L$ and the Minimal Sets of Scattering Data . . . 16

4 Spectral Properties of Lax Operators on Symmetric Spaces. Constant Boundary Conditions 20

4.1 Jost Solutions and Continuous Spectrum of $L$. . . . . . . . . . 21

4.2 Jost Solutions, Scattering Matrix and FAS . . . . . . . . . . . 23

4.3 The Resolvent of $L$ and Minimal Set of Scattering Data . . . . . . 28

5 Examples 29

5.1 Symmetric Spaces of A.III-Type and Vector NLSE . . . . . . . . 29

5.2 Symmetric Spaces of C.II and D.III Type . . . . . . . . . . . . 33

5.3 The Principal Minors and Fundamental Representations . . . . . . 35

6 Discussion

\section{Introduction}

The nonlinear Schrödinger equation (NLSE) with constant boundary conditions (CBC) is well known [18, 28, 29,31]. Here we address the so called multicomponent NLSE (MNLSE) with CBC whose dark soliton solutions [26] recently attracted attention with possible applications to Bose-Einstein condensates [22]. We start with the formulation of the vector NLSE with CBC:

$$
\mathrm{i} \frac{\partial \boldsymbol{q}}{\partial t}+\frac{\partial^{2} \boldsymbol{q}}{\partial x^{2}}-2\left(\boldsymbol{q}^{\dagger}, \boldsymbol{q}\right) \boldsymbol{q}(x, t)+\rho^{2} \boldsymbol{q}(x, t)+\left(\boldsymbol{q}_{ \pm}^{\dagger}, \boldsymbol{q}(x, t)\right) \boldsymbol{q}_{ \pm}=0
$$

Here $\boldsymbol{q}(x, t)$ is an $n$-component vector-valued function tending to the constant vectors $\boldsymbol{q}_{ \pm}$, i.e., $\lim _{x \rightarrow \pm \infty} \boldsymbol{q}(x, t)=\boldsymbol{q}_{ \pm}$and $\rho^{2}=\left(\boldsymbol{q}_{ \pm}^{\dagger}, \boldsymbol{q}_{ \pm}\right)$. The additional linear in $\boldsymbol{q}$ terms in (1) are used as a regularization which avoids the strong oscillations of $\boldsymbol{q}(x, t)$ in $t$ for $x \rightarrow \pm \infty$.

The equation (1) allows a Lax representation $[L(\lambda), M(\lambda)]=0$ which is similar to the one for the Manakov model [25]. The Lax operator $L$ is a special form of 
the generalized block-matrix Zakharov-Shabat system

$$
\begin{gathered}
L \psi(x, \lambda) \equiv \mathrm{i} \frac{\mathrm{d} \psi}{\mathrm{d} x}+q(x) \psi(x, \lambda)-\lambda J \psi(x, \lambda)=0 \\
q(x, t)=\left(\begin{array}{cc}
0 & \boldsymbol{q}^{T}(x, t) \\
-\boldsymbol{q}^{*}(x, t) & 0
\end{array}\right), \quad J=\left(\begin{array}{cc}
1 & 0 \\
0 & -\mathbb{1}_{n}
\end{array}\right) .
\end{gathered}
$$

All the considerations displayed below are valid for any choice of the block dimensions; in some of the specific examples we take $n=3$ for simplicity.

The $M$-operator is given by

$$
\begin{gathered}
M \psi \equiv \mathrm{i} \frac{\mathrm{d} \psi}{\mathrm{d} t}+\left(V_{0}(x, t)-V_{0,+}+2 \lambda q(x, t)-2 \lambda^{2} J\right) \psi(x, t, \lambda)=\psi(x, t, \lambda) C(\lambda) \\
V_{0}(x, t)=\left[\operatorname{ad}_{J}^{-1} q, q(x, t)\right]+2 \operatorname{iad}_{J}^{-1} q_{x}, \quad V_{0, \pm}=\lim _{x \rightarrow \pm \infty} V_{0}(x, t) .
\end{gathered}
$$

The formal expressions for the Lax pair are written down for the vector NLSE. However with minor modifications they can be made to hold true for several types of symmetric spaces: A.III, C.II, D.III and BD.I. Indeed, let us choose a slightly more general form of $L$. It is based on the choice of the simple Lie algebra $\mathfrak{g}$ and a Cartan involution that specifies the corresponding symmetric space. As it is well known [21] each symmetric space is a factor space $\mathfrak{G} / \mathfrak{H}$, where $\mathfrak{G}$ is the simple Lie group with Lie algebra $\mathfrak{g}$ and $\mathfrak{H}$ is a normal subgroup of $\mathfrak{G}$. In fact, $\mathfrak{H}$ is the invariant subspace of the Cartan involution (automorphism of second order). On algebraic level we can say that the Cartan involution splits the root system $\Delta$ of $\mathfrak{g}$ into two subsets

$$
\Delta=\Delta_{0} \cup \Delta_{1}, \quad \Delta^{+}=\Delta_{0}^{+} \cup \Delta_{1}^{+}
$$

according to the choice of $J \in \mathfrak{h}$ - constant real element of the Cartan subalgebra of $\mathfrak{g}$. The set of roots in $\Delta_{0}$ are such that $\alpha(J)=0$ for any $\alpha \in \Delta_{0}$, whereas $\beta_{1}(J)=\beta_{2}(J)>0$ for any two roots $\beta_{1}, \beta_{2} \in \Delta_{1}^{+}$. Below we will specify the sets $\Delta_{0}^{ \pm}, \Delta_{1}^{ \pm}$and $J$ for each of the relevant symmetric spaces.

The next step is to relate to each symmetric space a Lax operator of the form

$$
L \psi(x, \lambda) \equiv \mathrm{i} \frac{\mathrm{d} \psi}{\mathrm{d} x}+q(x, t) \psi(x, \lambda)-\lambda J \psi(x, \lambda)=0
$$

where the potential $q(x, t)(6)$ can always be represented as

$$
q(x, t)=[J, Q(x, t)] .
$$

Here $Q(x, t)$ is a generic element of $\mathfrak{g}$. In fact $q(x, t)$ provides local coordinates in the space tangent to $\mathfrak{G} / \mathfrak{H}$ at a given point and can be written down as:

$$
q(x, t)=\sum_{\alpha \in \Delta_{1}^{+}}\left(q_{\alpha}(x, t) E_{\alpha}+p_{\alpha}(x, t) E_{-\alpha}\right) .
$$


The Lax operator (2) is a particular case of (5) corresponding to a specific symmetric space of A.III type $\mathrm{SU}(n+1) / \mathrm{S}(\mathrm{U}(1) \times \mathrm{U}(n))$; its spectral properties for vanishing boundary conditions (VBC) were analyzed in [25].

The purpose of the present paper is to outline the spectral properties of $L$ for two classes of potentials:

i) $q(x, t)$ is a Schwartz-type function of $x$ and $t$ taking values in $\mathfrak{g} / \mathfrak{h}$.

ii) $q(x, t)$ is a smooth function of $x$ and $t$ taking values in $\mathfrak{g} / \mathfrak{h}$ and satisfying constant boundary conditions

$$
\lim _{x \rightarrow \pm \infty} q(x, t)=q_{ \pm}
$$

where $q_{ \pm}$are properly chosen constant elements of $\mathfrak{g} / \mathfrak{k}$.

iii) We also impose one more implicit condition on the potentials, namely we assume that $q(x, t)$ is such, that the corresponding Lax operator $L$ has a finite number of simple eigenvalues.

Next we will use these results for the analysis of the NLEE generated by these Lax operators.

In doing so we will need somewhat different approaches depending on the choice of $J$. The first one applies to $J$ 's given by equation (11) and satisfying the characteristic equation $J^{2}=\mathbb{1}$. Such choice of $J$ is relevant for symmetric spaces of types A.III, C.II and D.III in the Cartan classification. The second approach deals with $J$ 's of the form

$$
J=\left(\begin{array}{rlr}
1 & 0 & 0 \\
0 & 0_{n} & 0 \\
0 & 0 & -1
\end{array}\right)
$$

where $0_{n}$ is an $n \times n$ matrix whose matrix elements are all vanishing, i.e., the vanishing eigenvalue has multiplicity $n$. Such $J$ 's satisfy the characteristic equation $J^{n}\left(J^{2}-\mathbb{1}\right)=0$ and are relevant for the class of BD.I type symmetric spaces of the form $\mathrm{SO}(n+2) /(\mathrm{SO}(n) \otimes \mathrm{SO}(2))$. Such spaces exist both for odd and even values of $n$. The element $J$ inevitably has vanishing eigenvalues and is dual to the vector $e_{1}$.

Of course, in analyzing the spectral properties of the Lax operators we will use the typical (i.e., lowest dimensional) representations of the relevant algebras.

In the next Section 2 we provide some algebraic preliminaries for the symmetric spaces of type A.I, C.II, D.III, and for completeness, also for BD.I-type spaces. 
In Section 3 we outline the construction of the Jost solutions, scattering matrix and the fundamental analytic solutions (FAS) of $L$ for VBC. Most of the considerations are done in the typical representations of the corresponding simple Lie algebras. However these results can be generalized to any irreducible representation. In particular we propose a formulation of the minimal set of scattering data which is invariant with respect to the choice of the representation. In Section 4 we construct the Jost solutions, the scattering matrix and the FAS for the CBC case. Section 5 is dedicated to several examples for specific choices of the symmetric spaces of A.III, C.II and D.III-types.

\section{Algebraic Preliminaries}

In order to proceed with the symmetric spaces we mentioned above we will need to introduce their Cartan-Weyl basis in the typical representations and the corresponding Cartan involution definitions. In fact it will be sufficient to specify the sets of roots forming $\Delta_{0}^{+}$and $\Delta_{1}^{+}$along with the corresponding choice of the real constant element $J$.

First we start with the A.III series; the corresponding Lie algebras are $\mathfrak{g} \simeq \mathfrak{s l}(r+$ 1). Their set of positive roots is provided by

$$
\Delta^{+}=\left\{e_{i}-e_{j} ; 1 \leq i<j \leq r+1\right\}
$$

In fact the root space of $\mathfrak{s l}(r+1)$ is the $r$-dimensional subspace of $\mathbb{E}^{r+1}$ orthogonal to the vector $\vec{\varepsilon}_{0}=\sum_{k=1}^{r+1} e_{k}$. The Cartan-Weyl basis in the typical representation is given by

$$
H_{\alpha}=E_{i i}-E_{j, j}, \quad E_{\alpha}=E_{i j}, \quad E_{-\alpha}=E_{\alpha}^{T}, \quad 1 \leq i<j \leq r+1
$$

where $E_{i j}$ is an $r+1 \times r+1$ matrix with matrix elements $\left(E_{i j}\right)_{k l}=\delta_{i k} \delta_{j l}$. For the A.III type symmetric spaces we choose

$$
J=\left(\begin{array}{cc}
\mathbb{1}_{s} & 0 \\
0 & -\mathbb{1}_{s^{\prime}}
\end{array}\right), \quad s+s^{\prime}=r+1
$$

the element $J$ is dual to the vector $\sum_{k=1}^{s} e_{k}-\sum_{k=s+1}^{r+1} e_{k}+\left(s^{\prime}-s\right) /(r+1) \vec{\varepsilon}_{0}$ in the root space.

In what follows we will use slightly different definition of the orthogonal and symplectic Lie algebras, namely

$$
\mathfrak{g} \equiv\left\{X ; X+S_{0} X^{T} S_{0}^{-1}=0\right\}
$$


where

$$
\begin{array}{rlrl}
S_{0} & =\sum_{s=1}^{2 r+1}(-1)^{s+1} E_{s \bar{s}} & \text { for } & \mathfrak{g} \simeq \mathfrak{s o}(2 r+1) \\
S_{0} & =\sum_{s=1}^{r}(-1)^{s+1}\left(E_{s \bar{s}}-E_{\bar{s} s}\right) & \text { for } & \mathfrak{g} \simeq \mathfrak{s p}(2 r) \\
S_{0} & =\sum_{s=1}^{r}(-1)^{s+1}\left(E_{s \bar{s}}+E_{\bar{s} s}\right) & \text { for } \quad \mathfrak{g} \simeq \mathfrak{s o}(2 r) .
\end{array}
$$

If we denote by $N$ the dimension of the typical representation of the corresponding algebra then $\bar{s}=N+1-s$ and $E_{k s}$ are $N \times N$ matrices defined by $\left(E_{k s}\right)_{j l}=\delta_{k j} \delta_{s l}$. For $\mathfrak{g} \simeq \mathfrak{s o}(2 r+1)$ we have $N=2 r+1$; for $\mathfrak{g} \simeq \mathfrak{s o}(2 r)$ and $\mathfrak{g} \simeq \mathfrak{s p}(2 r)$ we have $N=2 r$.

Note that $S_{0}^{2}=\mathbb{1}$ for $\mathfrak{s o}(2 r+1)$ and $\mathfrak{s o}(2 r)$ and $S_{0}^{2}=-\mathbb{1}$ for $\mathfrak{s p}(2 r)$.

In what follows we denote by $e_{k}, k=1, \ldots, r$ the vectors forming an orthonormal basis in the root spaces $\mathbb{E}^{r}$ of the corresponding algebra. The above definitions of $\mathfrak{g}(12)$ has the advantage that the Cartan generators $H_{k}$ are diagonal. In particular, if $H_{k}$ is dual to $e_{k}$ it is given by

$$
H_{k}=E_{k k}-E_{\bar{k} \bar{k}}, \quad \bar{k}=N+1-k .
$$

The root systems of these algebras $\Delta=\Delta^{+} \cup\left(-\Delta^{+}\right)$are well known [21]:

$$
\begin{aligned}
& \Delta^{+}=\left\{e_{i}-e_{j} ; 1 \leq i<j \leq r+1\right\} \quad \text { for } \quad \mathfrak{s l}(r+1) \\
& \Delta^{+}=\left\{e_{i} \pm e_{j}, e_{j} ; 1 \leq i<j \leq r\right\} \quad \text { for } \quad \mathfrak{s o}(2 r+1) \\
& \Delta^{+}=\left\{e_{i} \pm e_{j} ; 1 \leq i<e_{j} \leq r\right\} \quad \text { for } \quad \mathfrak{s o}(2 r) \\
& \Delta^{+}=\left\{e_{i} \pm e_{j}, 2 e_{j} ; 1 \leq i<j \leq r\right\} \quad \text { for } \quad \mathfrak{s p}(2 r) \text {. }
\end{aligned}
$$

We also remind that to each element $J \in \mathfrak{h}$ there corresponds a dual vector $\vec{a}$ in the root space $\mathbb{E}^{r}$. We choose the element $J$ in the Lax operator in such a way that $\alpha(J)=(\alpha, \vec{a})>0$ for all roots $\alpha \in \Delta^{+}$.

The Cartan-Weyl basis in the typical representations are given by

$$
H_{k}=E_{k k}-\frac{1}{r+1} \mathbb{1}, \quad E_{e_{i}-e_{j}}=E_{i j}
$$

for $\mathfrak{s l}(r+1)$;

$$
\begin{aligned}
H_{k} & =E_{k k}-E_{\bar{k} \bar{k}}, & E_{e_{i}-e_{j}} & =E_{i j}-(-1)^{i+j} E_{\bar{j} \bar{i}} \\
E_{e_{i}+e_{j}} & =E_{i \bar{j}}-(-1)^{i+j} E_{j \bar{i}}, & E_{e_{j}} & =E_{r+1, j}-(-1)^{r+j} E_{\bar{j}, r+1}
\end{aligned}
$$


for $\mathfrak{s o}(2 r+1)$;

$$
\begin{aligned}
H_{k} & =E_{k k}-E_{\bar{k} \bar{k}}, & E_{e_{i}-e_{j}} & =E_{i j}-(-1)^{i+j} E_{\bar{j} \bar{i}} \\
E_{e_{i}+e_{j}} & =E_{i \bar{j}}+(-1)^{i+j} E_{j \bar{i}}, & E_{2 e_{j}} & =E_{j, \bar{j}}
\end{aligned}
$$

for $\mathfrak{s p}(2 r)$;

$$
H_{k}=E_{k k}-E_{\bar{k} \bar{k}}, E_{e_{i}-e_{j}}=E_{i j}-(-1)^{i+j} E_{\bar{j} \bar{i}}, E_{e_{i}+e_{j}}=E_{i \bar{j}}-(-1)^{i+j} E_{j \bar{i}}
$$

for $\mathfrak{s o}(2 r)$.

Most of the symmetric spaces which will used below are characterized by an element $J$ such that $J^{2}=\mathbb{1}$, see (11). $J$ is dual to the $\vec{a} \in \mathfrak{h}^{*}, J=\sum_{k=1}^{r} H_{k}$. Using $J$ we can split the set of positive roots into two subsets $\Delta^{+}=\Delta_{0}^{+} \cup \Delta_{1}^{+}$.

As we mentioned above, for the A.III-type symmetric spaces we choose $J$ to be dual to the vector $\vec{a}=\sum_{p=1}^{s} e_{p}-\sum_{p=s+1}^{r+1} e_{p}+\left(s^{\prime}-s\right) /(r+1) \vec{\varepsilon}_{0}$

$$
\begin{aligned}
& \Delta_{0}^{+}=\left\{e_{i}-e_{j} ; 1 \leq j \leq s \text { and } s+1 \leq i<j \leq N\right\} \\
& \Delta_{1}^{+}=\left\{e_{i}-e_{j} ; 1 \leq j \leq s \text { and } s+1 \leq i<j \leq N\right\}
\end{aligned}
$$

We will pay special attention to the case when $r+1=2 s$ and $s^{\prime}=s$. Such choice allows one also to treat two other classes of symmetric spaces: i) C.II with $\mathfrak{g} \simeq \mathfrak{s p}(2 r)$ and

$$
\Delta_{0}^{+}=\left\{e_{i}-e_{j}\right\}, \quad \Delta_{1}^{+}=\left\{2 e_{i} ; e_{i}+e_{j}\right\}, \quad 1 \leq i<j \leq r
$$

and also ii) D.III with $\mathfrak{g} \simeq \mathfrak{s o}(2 r)$ and

$$
\Delta_{0}^{+}=\left\{e_{i}-e_{j}\right\}, \quad \Delta_{1}^{+}=\left\{e_{i}+e_{j}\right\}, \quad 1 \leq i<j \leq r .
$$

The corresponding $J$ in both cases is dual to $\sum_{p=1}^{r} e_{p}$. For completeness we include here also the case of BD.I-type symmetric spaces which are of the form $\mathrm{SO}(n+2) /(\mathrm{SO}(n) \otimes \mathrm{SO}(2))$ and exist both for odd and even values of $n$. The element $J$ satisfies the characteristic equation $J^{3}-J=0$ and inevitably has vanishing eigenvalues, see equation (8). As a result we have two possibilities

$$
\begin{array}{ll}
\Delta_{0}^{+}=\left\{e_{1} \pm e_{j}, e_{1} ; 1 \leq j \leq r\right\}, & \Delta_{1}^{+}=\left\{e_{i} \pm e_{j}, e_{i} ; 2 \leq i<j \leq r\right\} \\
\Delta_{0}^{+}=\left\{e_{1} \pm e_{j} ; 1 \leq j \leq r\right\}, & \Delta_{1}^{+}=\left\{e_{i} \pm e_{j} ; 2 \leq i<j \leq r\right\}
\end{array}
$$

where the first line in equation (28) is valid for $n=2 r+1$ and the second one for $n=2 r$. 


\section{Spectral Properties of Lax Operators on Symmetric Spaces. Vanishing Boundary Conditions}

\subsection{Jost Solutions, Scattering Matrix and FAS}

The spectral theory of the Lax operators related to the symmetric spaces of A.III, C.II and D.III-types can be constructed along the same lines ${ }^{1}$. Their continuous spectrum for vanishing boundary conditions fills up the real axis of the complex $\lambda$-plane, see $[4,5,10,25]$; more recently the topic was covered in [2]. The corresponding Jost solutions and FAS are also constructed rather straightforwardly. In our considerations below we will use the lowest-dimensional nontrivial representation of the corresponding Lie algebras.

In our consideration $t$ plays the role of an additional parameter; for the sake of brevity the $t$-dependence is mostly suppressed. Condition iii) on page 4 can not be formulated as a set of explicit conditions on $q(x)$; its precise meaning will become clear below. The main tool here are the Jost solutions defined by their asymptotics at $x \rightarrow \pm \infty$

$$
\lim _{x \rightarrow \infty} \psi(x, \lambda) \mathrm{e}^{\mathrm{i} \lambda J x}=\mathbb{1}, \quad \lim _{x \rightarrow-\infty} \phi(x, \lambda) \mathrm{e}^{\mathrm{i} \lambda J x}=\mathbb{1} .
$$

Along with the Jost solutions we introduce

$$
\xi(x, \lambda)=\psi(x, \lambda) \mathrm{e}^{\mathrm{i} \lambda J x}, \quad \varphi(x, \lambda)=\phi(x, \lambda) \mathrm{e}^{\mathrm{i} \lambda J x}
$$

which satisfy the following linear integral equations

$$
\begin{aligned}
& \xi(x, \lambda)=\mathbb{1}+\mathrm{i} \int_{\infty}^{x} \mathrm{~d} y \mathrm{e}^{-\mathrm{i} \lambda J(x-y)} q(y) \xi(y, \lambda) \mathrm{e}^{\mathrm{i} \lambda J(x-y)} \\
& \varphi(x, \lambda)=\mathbb{1}+\mathrm{i} \int_{-\infty}^{x} \mathrm{~d} y \mathrm{e}^{-\mathrm{i} \lambda J(x-y)} q(y) \varphi(y, \lambda) \mathrm{e}^{\mathrm{i} \lambda J(x-y)} .
\end{aligned}
$$

These are Volterra type equations which, as is well known always have solutions, provided one can ensure the convergence of the integrals in the right hand side. For $\lambda$ real the exponential factors in (31) and (32) are just oscillating and the convergence is ensured by condition i) on page 4 .

Obviously the Jost solutions as whole can not be extended for $\operatorname{im} \lambda \neq 0$. However some of their columns can be extended for $\lambda \in \mathbb{C}_{+}$, others - for $\lambda \in \mathbb{C}_{-}$. Indeed, the equation (31) for the first $s$ columns of $\xi(x, \lambda)$ contains only the exponential

\footnotetext{
${ }^{1}$ Due to the fact that the element $J$ for the BD.I-type symmetric spaces has vanishing eigenvalues, the corresponding spectral problem requires additional considerations.
} 
factor $\mathrm{e}^{\mathrm{i} \lambda(x-y)}$ which falls off for $\operatorname{im} \lambda<0$. More precisely we can write down the Jost solutions $\psi(x, \lambda)$ and $\phi(x, \lambda)$ in the following block-matrix form

$$
\psi(x, \lambda)=\left(\left|\psi^{-}(x, \lambda)\right\rangle,\left|\psi^{+}(x, \lambda)\right\rangle\right), \quad \phi(x, \lambda)=\left(\left|\phi^{+}(x, \lambda)\right\rangle,\left|\phi^{-}(x, \lambda)\right\rangle\right)
$$

where the superscript + (respectively - ) shows that the corresponding blockmatrix allows analytic extension for $\lambda \in \mathbb{C}_{+}$(respectively $\lambda \in \mathbb{C}_{-}$).

Solving the direct scattering problem means given the potential $q(x)$ to find the scattering matrix $T(\lambda)$. By definition $T(\lambda)$ relates the two Jost solutions

$$
\phi(x, \lambda)=\psi(x, \lambda) T(\lambda), \quad T(\lambda)=\left(\begin{array}{cc}
\boldsymbol{a}^{+}(\lambda) & -\boldsymbol{b}^{-}(\lambda) \\
\boldsymbol{b}^{+}(\lambda) & \boldsymbol{a}^{-}(\lambda)
\end{array}\right)
$$

and has compatible block-matrix structure. In what follows we will need also the inverse of the scattering matrix

$$
\psi(x, \lambda)=\phi(x, \lambda) \hat{T}(\lambda), \quad \hat{T}(\lambda) \equiv\left(\begin{array}{cc}
c^{-}(\lambda) & \mathrm{d}^{-}(\lambda) \\
-\mathrm{d}^{+}(\lambda) & \boldsymbol{c}^{+}(\lambda)
\end{array}\right)
$$

where

$$
\begin{aligned}
& \boldsymbol{c}^{-}(\lambda)=\hat{\boldsymbol{a}}^{+}(\lambda)\left(\mathbb{1}+\rho^{-} \rho^{+}\right)^{-1}=\left(\mathbb{1}+\tau^{+} \tau^{-}\right)^{-1} \hat{\boldsymbol{a}}^{+}(\lambda) \\
& \mathrm{d}^{-}(\lambda)=\hat{\boldsymbol{a}}^{+}(\lambda) \rho^{-}(\lambda)\left(\mathbb{1}+\rho^{+} \rho^{-}\right)^{-1}=\left(\mathbb{1}+\tau^{+} \tau^{-}\right)^{-1} \tau^{+}(\lambda) \hat{\boldsymbol{a}}^{-}(\lambda) \\
& \boldsymbol{c}^{+}(\lambda)=\hat{\boldsymbol{a}}^{-}(\lambda)\left(\mathbb{1}+\rho^{+} \rho^{-}\right)^{-1}=\left(\mathbb{1}+\tau^{-} \tau^{+}\right)^{-1} \hat{\boldsymbol{a}}^{-}(\lambda) \\
& \mathrm{d}^{+}(\lambda)=\hat{\boldsymbol{a}}^{-}(\lambda) \rho^{+}(\lambda)\left(\mathbb{1}+\rho^{-} \rho^{+}\right)^{-1}=\left(\mathbb{1}+\tau^{-} \tau^{+}\right)^{-1} \tau^{-}(\lambda) \hat{\boldsymbol{a}}^{+}(\lambda) .
\end{aligned}
$$

The diagonal blocks of both $T(\lambda)$ and $\hat{T}(\lambda)$ allow analytic continuation off the real axis, namely $\boldsymbol{a}^{+}(\lambda), \boldsymbol{c}^{+}(\lambda)$ are analytic functions of $\lambda$ for $\lambda \in \mathbb{C}_{ \pm}$, while $\boldsymbol{a}^{-}(\lambda), \boldsymbol{c}^{-}(\lambda)$ are analytic functions of $\lambda$ for $\lambda \in \mathbb{C}_{ \pm}$.

By $\rho^{ \pm}(\lambda)$ and $\tau^{ \pm}(\lambda)$ above we have denoted the multicomponent generalizations of the reflection coefficients $[4,5]$, generalizing the ones for the scalar case, see $[1,15,23]$

$$
\rho^{ \pm}(\lambda)=\boldsymbol{b}^{ \pm} \hat{\boldsymbol{a}}^{ \pm}(\lambda)=\hat{\boldsymbol{c}}^{ \pm} \mathrm{d}^{ \pm}(\lambda), \quad \tau^{ \pm}(\lambda)=\hat{\boldsymbol{a}}^{ \pm} \boldsymbol{b}^{\mp}(\lambda)=\mathrm{d}^{\mp} \hat{\boldsymbol{c}}^{ \pm}(\lambda) .
$$

We will need also the asymptotics for $\lambda \rightarrow \infty$

$$
\begin{gathered}
\lim _{\lambda \rightarrow-\infty} \phi(x, \lambda) \mathrm{e}^{\mathrm{i} \lambda J x}=\lim _{\lambda \rightarrow \infty} \psi(x, \lambda) \mathrm{e}^{\mathrm{i} \lambda J x}=\mathbb{1}, \quad \lim _{\lambda \rightarrow \infty} T(\lambda)=\mathbb{1} \\
\lim _{\lambda \rightarrow \infty} \boldsymbol{a}^{+}(\lambda)=\lim _{\lambda \rightarrow \infty} \boldsymbol{c}^{-}(\lambda)=\mathbb{1}, \quad \lim _{\lambda \rightarrow \infty} \boldsymbol{a}^{-}(\lambda)=\lim _{\lambda \rightarrow \infty} \boldsymbol{c}^{+}(\lambda)=\mathbb{1} .
\end{gathered}
$$


The inverse to the Jost solutions $\hat{\psi}(x, \lambda)$ and $\hat{\phi}(x, \lambda)$ are solutions to

$$
\mathrm{i} \frac{\mathrm{d} \hat{\psi}}{\mathrm{d} x}-\hat{\psi}(x, \lambda)(q(x)-\lambda J)=0
$$

satisfying the conditions

$$
\lim _{x \rightarrow \infty} \mathrm{e}^{-\mathrm{i} \lambda J x} \hat{\psi}(x, \lambda)=\mathbb{1}, \quad \lim _{x \rightarrow-\infty} \mathrm{e}^{-\mathrm{i} \lambda J x} \hat{\phi}(x, \lambda)=\mathbb{1} .
$$

Now it is the collections of rows of $\hat{\psi}(x, \lambda)$ and $\hat{\phi}(x, \lambda)$ that possess analytic properties in $\lambda$

$$
\hat{\psi}(x, \lambda)=\left(\begin{array}{l}
\left\langle\hat{\psi}^{+}(x, \lambda)\right| \\
\left\langle\hat{\psi}^{-}(x, \lambda)\right|
\end{array}\right), \quad \hat{\phi}(x, \lambda)=\left(\begin{array}{c}
\left\langle\hat{\phi}^{-}(x, \lambda)\right| \\
\left\langle\hat{\phi}^{+}(x, \lambda)\right|
\end{array}\right) .
$$

Just like the Jost solutions, their inverse (41) are solutions to linear equations (39) with regular boundary conditions (40). Therefore, they can have no singularities in their regions of analyticity. The same holds true also for the scattering matrix $T(\lambda)=\hat{\psi}(x, \lambda) \phi(x, \lambda)$ and its inverse $\hat{T}(\lambda)=\hat{\phi}(x, \lambda) \psi(x, \lambda)$, i.e.,

$$
\boldsymbol{a}^{+}(\lambda)=\left\langle\hat{\psi}^{+}(x, \lambda) \mid \phi^{+}(x, \lambda)\right\rangle, \quad \boldsymbol{a}^{-}(\lambda)=\left\langle\hat{\psi}^{-}(x, \lambda) \mid \phi^{-}(x, \lambda)\right\rangle
$$

as well as

$$
\boldsymbol{c}^{+}(\lambda)=\left\langle\hat{\phi}^{+}(x, \lambda) \mid \psi^{+}(x, \lambda)\right\rangle, \quad \boldsymbol{c}^{-}(\lambda)=\left\langle\hat{\phi}^{-}(x, \lambda) \mid \psi^{-}(x, \lambda)\right\rangle
$$

are analytic for $\lambda \in \mathbb{C}_{ \pm}$and have no singularities in their regions of analyticity. However they may become degenerate (i.e., their determinants may vanish) for some values $\lambda_{j}^{ \pm} \in \mathbb{C}_{ \pm}$of $\lambda$. Below we analyze the structure of these degeneracies.

\subsection{The Fundamental Analytic Solutions}

The next step is to construct the fundamental analytic solutions of $L$. In our case this is done simply by combining the blocks of Jost solutions with the same analytic properties

$$
\begin{aligned}
& \chi^{+}(x, \lambda)=\phi(x, \lambda) S^{+}(\lambda)=\psi(x, \lambda) T^{-}(\lambda) D^{+}(\lambda) \\
& \chi^{-}(x, \lambda)=\phi(x, \lambda) S^{-}(\lambda)=\psi(x, \lambda) T^{+}(\lambda) D^{-}(\lambda)
\end{aligned}
$$

where the block-triangular functions $\mathrm{S}^{ \pm}(\lambda)$ and $\boldsymbol{T}^{ \pm}(\lambda)$ are given by

$$
\begin{aligned}
S^{+}(\lambda) & =\left(\begin{array}{cc}
\mathbb{1} & \tau^{+}(\lambda) \\
0 & \mathbb{1}
\end{array}\right), & \boldsymbol{T}^{-}(\lambda) & =\left(\begin{array}{cc}
\mathbb{1} & 0 \\
\rho^{+}(\lambda) & \mathbb{1}
\end{array}\right) \\
S^{-}(\lambda) & =\left(\begin{array}{cc}
\mathbb{1} & 0 \\
-\tau^{-}(\lambda) & \mathbb{1}
\end{array}\right), & T^{+}(\lambda) & =\left(\begin{array}{cc}
\mathbb{1} & -\rho^{-}(\lambda) \\
0 & \mathbb{1}
\end{array}\right) \\
D^{+}(\lambda) & =\left(\begin{array}{cc}
\boldsymbol{a}^{+}(\lambda) & 0 \\
0 & \hat{\boldsymbol{c}}^{+}(\lambda)
\end{array}\right), & D^{-}(\lambda) & =\left(\begin{array}{cc}
\hat{\boldsymbol{c}}^{-}(\lambda) & 0 \\
0 & \boldsymbol{a}^{-}(\lambda)
\end{array}\right)
\end{aligned}
$$


and $\rho^{ \pm}(\lambda)$ and $\tau^{ \pm}(\lambda)$ are given by equation (37).

The factors $S^{ \pm}(\lambda), T^{ \pm}(\lambda)$ and $D^{ \pm}(\lambda)$ are related to the scattering matrix $T(\lambda)$ and its inverse $\hat{T}(\lambda)$ by

$$
\begin{aligned}
& T(\lambda)=T^{-}(\lambda) D^{+}(\lambda) \hat{S}^{+}(\lambda)=T^{+}(\lambda) D^{-}(\lambda) \hat{S}^{-}(\lambda) \\
& \hat{T}(\lambda)=S^{+}(\lambda) \hat{D}^{+}(\lambda) \hat{T}^{-}(\lambda)=S^{-}(\lambda) \hat{D}^{-}(\lambda) \hat{T}^{+}(\lambda) .
\end{aligned}
$$

In other words equation (46) and can be viewed as generalized Gauss decompositions (see [21]) of $T(\lambda)$ and its inverse.

The relations between $\boldsymbol{c}^{ \pm}(\lambda), \mathrm{d}^{ \pm}(\lambda)$ and $\boldsymbol{a}^{ \pm}(\lambda), \boldsymbol{b}^{ \pm}(\lambda)$ in equation (36) ensure that equations (46) become identities. From equations (44), (45) we derive

$$
\begin{aligned}
& \chi^{+}(x, \lambda)=\chi^{-}(x, \lambda) G_{0}(\lambda), \quad \chi^{-}(x, \lambda)=\chi^{+}(x, \lambda) \hat{G}_{0}(\lambda) \\
& G_{0}(\lambda)=\hat{S}^{-} S^{+}(\lambda)=\left(\begin{array}{cc}
\mathbb{1} & \tau^{+}(\lambda) \\
\tau^{-}(\lambda) & \mathbb{1}+\tau^{-}(\lambda) \tau^{+}(\lambda)
\end{array}\right) \\
& \hat{G}_{0}(\lambda)=\hat{S}^{+} S^{-}(\lambda)=\left(\begin{array}{cc}
\mathbb{1}+\tau^{+}(\lambda) \tau^{-}(\lambda) & \tau^{+}(\lambda) \\
-\tau^{-}(\lambda) & \mathbb{1}
\end{array}\right)
\end{aligned}
$$

valid for $\lambda \in \mathbb{R}$, where the block-triangular factors $S^{+}(\lambda)$ and $S^{-}(\lambda)$ are given by equation (45).

Note that the block-diagonal factors $D^{+}(\lambda)$ and $D^{-}(\lambda)$ are matrix-valued analytic functions for $\lambda \in \mathbb{C}_{ \pm}$, whereas $S^{ \pm}(\lambda)$ and $T^{ \pm}(\lambda)$ are defined only for real $\lambda \in \mathbb{R}$. Another well known fact about the FAS $\chi^{ \pm}(x, \lambda)$ concerns their asymptotic behavior for $\lambda \rightarrow \pm \infty$, namely, if we introduce

$$
X^{ \pm}(x, \lambda)=\chi^{ \pm}(x, \lambda) \mathrm{e}^{\mathrm{i} \lambda J x}
$$

then

$$
\lim _{\lambda \rightarrow \infty} X^{ \pm}(x, \lambda)=\mathbb{1} .
$$

With these notations equation (47) can be rewritten in the form

$$
X^{+}(x, \lambda)=X^{-}(x, \lambda) G(x, \lambda), \quad \lambda \in \mathbb{R}
$$

where

$$
G(x, \lambda)=\mathrm{e}^{-\mathrm{i} \lambda J x} G_{0}(\lambda) \mathrm{e}^{\mathrm{i} \lambda J x}=\left(\begin{array}{cc}
\mathbb{1} & \tau^{+}(\lambda) \mathrm{e}^{-2 \mathrm{i} \lambda x} \\
\tau^{-}(\lambda) \mathrm{e}^{2 \mathrm{i} \lambda x} & \mathbb{1}+\tau^{-}(\lambda) \tau^{+}(\lambda)
\end{array}\right) .
$$

From our considerations if $\chi^{ \pm}(x, \lambda)$ are the FAS of the Lax operator $L$ constructed above, then $X^{ \pm}(x, \lambda)$ will satisfy equation (52). The inverse is also true. Indeed, 
suppose we can construct two matrix-valued functions $X^{ \pm}(x, \lambda)$ which are analytic for $\lambda \in \mathbb{C}_{ \pm}$respectively, and which satisfy equations (52) and (51). Then one can show $[11,30,32]$ that $X^{ \pm}(x, \lambda)$ satisfy the equation

$$
\mathrm{i} \frac{\mathrm{d} X^{ \pm}}{\mathrm{d} x}+q(x) X^{ \pm}(x, \lambda)-\lambda\left[J, X^{ \pm}(x, \lambda)\right]=0
$$

and using the relation (50) we can recover the FAS of $L$. This important fact, along with the construction of the FAS for generalized Zakharov-Shabat systems has been discovered by Shabat [27].

Equation (52) is known as a Riemann-Hilbert problem (RHP). The additional constraint given by equation (51) is known as a canonical normalization of the RHP. In the derivations that follow the analyticity properties of $X^{ \pm}(x, \lambda)$ for $\lambda \in \mathbb{C}_{ \pm}$ and equation (52) will play crucial role.

Definition 1. We will say that $X^{ \pm}(x, \lambda)$ are regular solution to the RHP with canonical normalization if they have no singularities or zeroes in their regions of analyticity.

Then the following theorem holds.

Theorem 2. The RHP (52) has unique regular solution with canonical normalization condition (51).

Proof: Let us assume that along with $X^{ \pm}(x, \lambda)$ we have a second regular solution $X_{(1)}^{ \pm}(x, \lambda)$ for the same RHP, and let $Y^{ \pm}(x, \lambda)=X_{(1)}^{ \pm}(x, \lambda) \hat{X}^{ \pm}(x, \lambda)$. From equation (52) it is obvious that $Y^{+}(x, \lambda)=Y^{-}(x, \lambda)$ for $\lambda \in \mathbb{R}$ and also $\lim _{\lambda \rightarrow \infty} Y^{ \pm}(x, \lambda)=\mathbb{1}$. Since both solutions $X^{ \pm}(x, \lambda)$ and $X_{(1)}^{ \pm}(x, \lambda)$ are regular, then $Y^{ \pm}(x, \lambda)$ have neither zeroes nor singularities anywhere in the complex plane. Thus, from Liouville theorem there follows that $Y^{ \pm}(x, \lambda)=\mathbb{1}$, or

$$
X_{(1)}^{ \pm}(x, \lambda)=X^{ \pm}(x, \lambda) \text {. }
$$

The theorem is proved.

In what follows we will find out that the RHP allows nontrivial singular solutions, whose zeroes and singularities are located on the discrete spectrum of $L$. For the sake of simplicity in what follows we will reformulate the condition iii) on page 4 as follows

iii') Let the potential $q(x)$ of the operator $L$ be such that the corresponding FAS $X^{ \pm}(x, \lambda)$ have finite number of simple zeroes and singularities, located at the positions $\lambda_{k}^{ \pm} \in \mathbb{C}_{ \pm}, k=1, \ldots, N$. 
Remark 3. We will denote the set of the points $\lambda_{k}^{ \pm}$by $\mathfrak{S}$. As we will see below, each of these points is a discrete eigenvalue of $L$.

Note that the Jost solutions have no singularities, therefore singularities and zeroes of $X^{ \pm}(x, \lambda)$ can come up only if the matrices $\boldsymbol{a}^{ \pm}(\lambda), \boldsymbol{c}^{ \pm}(\lambda)$ become degenerate and, as a result, their inverse $\hat{\boldsymbol{a}}^{ \pm}(\lambda), \hat{\boldsymbol{c}}^{ \pm}(\lambda)$ acquire singularities. By simple zeroes and singularities in condition iii $^{\prime}$ ) above we mean that $\boldsymbol{a}^{ \pm}(\lambda), \boldsymbol{c}^{ \pm}(\lambda)$ and their inverse $\hat{\boldsymbol{a}}^{ \pm}(\lambda), \hat{\boldsymbol{c}}^{ \pm}(\lambda)$ have the following behavior in the vicinity of $\lambda_{k}^{ \pm}$

$$
\begin{aligned}
& \boldsymbol{a}^{ \pm}(\lambda)=\boldsymbol{a}_{k}^{ \pm}+\left(\lambda-\lambda_{k}^{ \pm}\right) \dot{\boldsymbol{a}}_{k}^{ \pm}+\mathcal{O}\left(\left(\lambda-\lambda_{k}^{ \pm}\right)^{2}\right) \\
& \boldsymbol{c}^{ \pm}(\lambda)=\boldsymbol{c}_{k}^{ \pm}+\left(\lambda-\lambda_{k}^{ \pm}\right) \dot{\boldsymbol{c}}_{k}^{ \pm}+\mathcal{O}\left(\left(\lambda-\lambda_{k}^{ \pm}\right)^{2}\right) \\
& \hat{\boldsymbol{a}}^{ \pm}(\lambda)=\frac{\hat{\boldsymbol{a}}_{k}^{ \pm}}{\lambda-\lambda_{k}^{ \pm}}+\hat{\dot{\boldsymbol{a}}}_{k}^{ \pm}+\mathcal{O}\left(\left(\lambda-\lambda_{k}^{ \pm}\right)\right) \\
& \hat{\boldsymbol{c}}^{ \pm}(\lambda)=\frac{\hat{\boldsymbol{c}}_{k}^{ \pm}}{\lambda-\lambda_{k}^{ \pm}}+\hat{\boldsymbol{c}}_{k}^{ \pm}+\mathcal{O}\left(\left(\lambda-\lambda_{k}^{ \pm}\right)\right) .
\end{aligned}
$$

Obviously, since we must have $\boldsymbol{a}^{ \pm}(\lambda) \hat{\boldsymbol{a}}^{ \pm}(\lambda)=\mathbb{1}$ for all $\lambda \in \mathbb{C}_{ \pm}$the matrices $\boldsymbol{a}_{k}^{ \pm}, \dot{\boldsymbol{a}}_{k}^{ \pm}, \hat{\boldsymbol{a}}_{k}^{ \pm}, \hat{\dot{\boldsymbol{a}}}_{k}^{ \pm}$must satisfy

$$
\boldsymbol{a}_{k}^{ \pm} \hat{\dot{\boldsymbol{a}}}_{k}^{ \pm}=0, \quad \hat{\boldsymbol{a}}_{k}^{ \pm} \dot{\boldsymbol{a}}_{k}^{ \pm}+\hat{\dot{\boldsymbol{a}}}_{k}^{ \pm} \boldsymbol{a}_{k}^{ \pm}=\mathbb{1}
$$

and similarly for the coefficients of $\boldsymbol{c}^{ \pm}(\lambda), \hat{\boldsymbol{c}}^{ \pm}(\lambda)$

$$
\boldsymbol{c}_{k}^{ \pm} \hat{\dot{c}}_{k}^{ \pm}=0, \quad \hat{\boldsymbol{c}}_{k}^{ \pm} \dot{\boldsymbol{c}}_{k}^{ \pm}+\hat{\dot{\boldsymbol{c}}}_{k}^{ \pm} \boldsymbol{c}_{k}^{ \pm}=\mathbb{1}
$$

Remark 4. A good tool for treating the scattering data related to the discrete spectrum of $L$ is based on the following. Consider first potentials on finite support. For such potentials not only the Jost solutions but also the scattering matrix elements allow analytic extension to the whole complex $\lambda$-plane. In that case the constant matrices $\boldsymbol{b}_{k}^{ \pm}, \mathrm{d}_{k}^{ \pm}$will be just the values of the functions $\boldsymbol{b}^{ \pm}(\lambda), \mathrm{d}^{ \pm}(\lambda)$ for $\lambda=\lambda_{k}^{ \pm}$:

$$
\boldsymbol{b}_{k}^{ \pm}=\left.\boldsymbol{b}^{ \pm}(\lambda)\right|_{\lambda=\lambda_{k}^{ \pm}}, \quad \mathrm{d}_{k}^{ \pm}=\left.\mathrm{d}^{ \pm}(\lambda)\right|_{\lambda=\lambda_{k}^{\mp}}
$$

Next one is to extend the support of the potential to the infinite line. This limit substantially changes the picture and the functions $\boldsymbol{b}^{ \pm}(\lambda), \mathrm{d}^{ \pm}(\lambda)$ can no more be extended outside $\lambda \in \mathbb{R}$. Therefore, in this limit $\boldsymbol{b}_{k}^{ \pm}, \mathrm{d}_{k}^{ \pm}$must be understood just as appropriate matrix-valued constants. 


\subsection{The FAS and Higher Representations}

Here we briefly outline the construction of the FAS for higher irreducible representations of $\mathfrak{g}$. To this end we will make use of the Cartan-Weyl basis and the possibility to represent the Gauss factors $S^{ \pm}(\lambda)$ and $T^{ \pm}(\lambda)$ in the form

$$
S^{ \pm}=\exp \left( \pm \sum_{\alpha \in \Delta_{1}^{+}} \tau_{\alpha}^{ \pm}(\lambda) E_{ \pm \alpha}\right), \quad T^{ \pm}=\exp \left(\mp \sum_{\alpha \in \Delta_{1}^{+}} \rho_{\alpha}^{\mp}(\lambda) E_{ \pm \alpha}\right) .
$$

The first step will be to interrelate the coefficients $\tau_{\alpha}^{\mp}(\lambda), \rho_{\alpha}^{\mp}(\lambda)$ to the matrix elements of $\tau^{ \pm}(\lambda), \rho^{ \pm}(\lambda)$ in the typical representations of $\mathfrak{g}$.

To this end we calculate:

$$
\begin{aligned}
& \hat{S}^{ \pm} J S^{ \pm}(\lambda)=J+2 \sum_{\alpha \in \Delta_{1}^{+}} \tau_{\alpha}^{ \pm}(\lambda) E_{ \pm \alpha} \\
& \hat{T}^{ \pm} J T^{ \pm}(\lambda)=J+2 \sum_{\alpha \in \Delta_{1}^{+}} \rho_{\alpha}^{\mp}(\lambda) E_{ \pm \alpha} .
\end{aligned}
$$

Here we made use of the specific property of the symmetric spaces, namely for any two roots $\beta_{1}$ and $\beta_{2}$ belonging to $\Delta_{1}^{+}$their sum $\beta_{1}+\beta_{2} \notin \Delta$ is not a root.

Thus we derive the following, invariant with respect to the choice of the representation expressions for the coefficients $\tau_{\alpha}^{ \pm}(\lambda), \rho_{\alpha}^{ \pm}(\lambda)$

$$
\tau_{\alpha}^{ \pm}(\lambda)=\frac{\left\langle\hat{S}^{ \pm} J S^{ \pm}(\lambda), E_{ \pm \alpha}\right\rangle}{\left\langle E_{\alpha}, E_{-\alpha}\right\rangle}, \quad \rho_{\alpha}^{\mp}(\lambda)=\frac{\left\langle\hat{T}^{ \pm} J T^{ \pm}(\lambda), E_{ \pm \alpha}\right\rangle}{\left\langle E_{\alpha}, E_{-\alpha}\right\rangle}
$$

where $\langle X, Y\rangle$ is the Killing form evaluated for $X, Y \in \mathfrak{g}$. These formulae hold for any choice of the irreducible representation of $\mathfrak{g}$.

It is also important to derive similar expressions for the coefficients of the variations $\delta \tau_{\alpha}^{\mp}(\lambda), \delta \rho_{\alpha}^{\mp}(\lambda)$. Using similar arguments as above we get

$$
\hat{S}^{ \pm} \delta S^{ \pm}(\lambda)= \pm \sum_{\alpha \in \Delta_{1}^{+}} \delta \tau_{\alpha}^{ \pm}(\lambda) E_{ \pm \alpha}, \quad \hat{T}^{ \pm} \delta T^{ \pm}(\lambda)=\mp \sum_{\alpha \in \Delta_{1}^{+}} \delta \rho_{\alpha}^{\mp}(\lambda) E_{ \pm \alpha} .
$$

Therefore,

$$
\left.\delta \tau_{\alpha}^{ \pm}(\lambda)= \pm \frac{\left\langle\hat{S}^{ \pm} \delta S^{ \pm}(\lambda), E_{ \pm \alpha}\right\rangle}{\left\langle E_{\alpha}, E_{-\alpha}\right\rangle}, \quad \delta \rho_{\alpha}^{\mp}(\lambda)=\mp \frac{\left\langle\hat{T}^{ \pm} \delta T^{ \pm}(\lambda), E_{ \pm \alpha}\right\rangle}{\left\langle E_{\alpha}, E_{-\alpha}\right\rangle}\right] .
$$

Such expressions are important in deriving the explicit of the action-angle variables for the corresponding MNLSE, as well as in analyzing their gauge equivalent nonlinear evolution equations of Heisenberg ferromagnet type. 


\subsection{Typical Reductions}

The typical reductions of the Lax pair and, as a consequence, the typical reductions of the MNLSE are of the form

$$
C_{0} U^{\dagger}\left(x, t, \lambda^{*}\right) \hat{C}_{0}=U(x, t, \lambda), \quad U(x, t, \lambda)=q(x, t)-\lambda J
$$

where $C_{0}^{2}=\mathbb{1}$. Two possible choices for $C_{0}$ are most important

$$
\begin{aligned}
& \text { a) } \quad C_{0}=\mathbb{1}, \quad \text { i.e., } \quad q=q^{\dagger} \\
& \text { b) } \quad C_{0}=J, \quad \text { i.e., } \quad q=J q^{\dagger} J .
\end{aligned}
$$

Of course, for symmetric spaces of rank higher than 1 one can choose as $C_{0}$ also Weyl group element, corresponding to a reflection with respect to a root $\alpha_{1}$ such, that $\alpha_{1}(J)=0$. Such reductions have been analyzed in [14].

Each of the above mentioned reductions imposes restriction on the scattering matrix $T(\lambda)$ and the scattering data as follows

$$
C_{0} T^{\dagger}\left(t, \lambda^{*}\right) \hat{C}_{0}=\hat{T}(t, \lambda)
$$

or, in terms of the block-components

$$
\begin{aligned}
\left(\boldsymbol{a}^{+}\left(\lambda^{*}\right)\right)^{\dagger} & =\boldsymbol{c}^{-}(\lambda), & & \left(\boldsymbol{a}^{-}\left(\lambda^{*}\right)\right)^{\dagger}=\boldsymbol{c}^{+}(\lambda) \\
\left(\boldsymbol{b}^{+}\left(\lambda^{*}\right)\right)^{\dagger} & =\varepsilon \mathrm{d}^{-}(\lambda), & & \left(\boldsymbol{b}^{-}\left(\lambda^{*}\right)\right)^{\dagger}=\varepsilon \mathrm{d}^{+}(\lambda) \\
\left(\rho^{-}\left(\lambda^{*}\right)\right)^{\dagger} & =\varepsilon \rho^{+}(\lambda), & & \left(\tau^{-}\left(\lambda^{*}\right)\right)^{\dagger}=\varepsilon \tau^{+}(\lambda)
\end{aligned}
$$

where $\varepsilon=1$ in case a) and $\varepsilon=-1$ in case b). From (56) there follows that

$$
\lambda_{k}^{+}=\left(\lambda_{k}^{-}\right)^{*}
$$

besides, relations similar to (68) hold true also for the coefficients $\boldsymbol{a}_{k}^{ \pm}, \boldsymbol{b}_{k}^{ \pm}, \boldsymbol{c}_{k}^{ \pm}, \mathrm{d}_{k}^{ \pm}$

$$
\begin{aligned}
\left(\boldsymbol{a}_{k}^{+}\right)^{\dagger} & =\boldsymbol{c}_{k}^{-}, & \left(\boldsymbol{a}_{k}^{-}\right)^{\dagger} & =\boldsymbol{c}_{k}^{+} \\
\left(\boldsymbol{b}_{k}^{+}\right)^{\dagger} & =\varepsilon \mathrm{d}_{k}^{-}, & \left(\boldsymbol{b}_{k}^{-}\right)^{\dagger} & =\varepsilon \mathrm{d}_{k}^{+} \\
\left(\rho_{k}^{-}\right)^{\dagger} & =\varepsilon \rho_{k}^{+}, & \left(\tau_{k}^{-}\right)^{\dagger} & =\varepsilon \tau_{k}^{+} \\
\rho_{k}^{ \pm}=\boldsymbol{b}_{k}^{ \pm} \hat{\boldsymbol{a}}_{k}^{ \pm} & =\hat{\boldsymbol{c}}_{k}^{ \pm} \mathrm{d}_{k}^{ \pm}, & \tau_{k}^{-}=\mathrm{d}_{k}^{\mp} \hat{\boldsymbol{c}}_{k}^{ \pm} & =\hat{\boldsymbol{a}}_{k}^{ \pm} \boldsymbol{b}_{k}^{\mp} .
\end{aligned}
$$

We provide also the corresponding constraints which the typical reductions impose on the coefficients $\tau_{\alpha}^{ \pm}(\lambda)$ and $\rho_{\alpha}^{ \pm}(\lambda)$

$$
\tau_{\alpha}^{-}(\lambda)=\varepsilon\left(\tau_{\alpha}^{+}\left(\lambda^{*}\right)\right)^{*}, \quad \rho_{\alpha}^{-}(\lambda)=\varepsilon\left(\rho_{\alpha}^{+}\left(\lambda^{*}\right)\right)^{*} .
$$


Remark 5. Reduction (66a) is typical for the MNLSE with vanishing boundary conditions (VBC). An important property of the other reduction (66b), which is typical for the MNLSE with CBC, consist in the fact that the Lax equation (2) becomes equivalent to a self-adjoint eigenvalue problem

$$
\mathfrak{L} \psi(x, \lambda) \equiv \mathrm{i} J \frac{\mathrm{d} \psi}{\mathrm{d} x}+J q(x) \psi(x, \lambda)=\lambda \psi(x, \lambda)
$$

since $J q(x, t)=(J q(x, t))^{\dagger}$. The spectrum of such operators must lie on the real $\lambda$-axis. However, for VBC the real $\lambda$-axis is already filled up by the continuous spectrum of $L$ which leaves "no space" for discrete eigenvalues of $L$. That is why the corresponding MNLSE do not have soliton solutions. When we go to $\mathrm{CBC}$ the situation changes. As we shall see below, the continuous spectrum of $L$ with $\mathrm{CBC}$ has lacunae on the real $\lambda$-axis and they admit real discrete eigenvalues inside these lacunae. The corresponding soliton solutions of the MNLSE with CBC are known as dark solitons.

\subsection{The Resolvent of $L$ and the Minimal Sets of Scattering Data}

The FAS allow one to construct explicitly the resolvent $\boldsymbol{R}_{\lambda}$ of $L$. The resolvent is an integral operator acting in the space of vector-valued functions $\vec{f}(x)$ by

$$
\left(\boldsymbol{R}_{\lambda} \vec{f}\right)(x)=\int_{-\infty}^{\infty} \mathrm{d} y R^{ \pm}(x, y, \lambda) \vec{f}(y)
$$

where the superscript + (respectively - ) corresponds to $\lambda \in \mathbb{C}_{+}$(respectively $\left.\lambda \in \mathbb{C}_{-}\right)$. For real values of $\lambda$ we use

$$
R(x, y, \lambda)=\frac{1}{2}\left(R^{+}(x, y, \lambda)+R^{-}(x, y, \lambda)\right), \quad \lambda \in \mathbb{R} .
$$

The kernel of the resolvent is provided by

$$
R^{ \pm}(x, y, \lambda)=\frac{1}{\mathrm{i}} \chi^{ \pm}(x, \lambda) \Theta^{ \pm}(x-y) \hat{\chi}^{ \pm}(y, \lambda), \quad \lambda \in \mathbb{C}_{ \pm}
$$

where

$$
\begin{aligned}
& \Theta^{+}(x-y)=\left(\begin{array}{cc}
-\theta(y-x) \mathbb{1}_{s} & 0 \\
0 & \theta(x-y) \mathbb{1}_{s^{\prime}}
\end{array}\right) \\
& \Theta^{-}(x-y)=\left(\begin{array}{cc}
\theta(x-y) \mathbb{1}_{s} & 0 \\
0 & -\theta(y-x) \mathbb{1}_{s^{\prime}}
\end{array}\right) .
\end{aligned}
$$

Skipping the details, we formulate the properties of $\boldsymbol{R}_{\lambda}$ (see $[9,11,12]$ ). 
Theorem 6. Let $q(x)$ satisfy conditions i) and $\left.\mathrm{iii}^{\prime}\right)$ and let $\lambda_{j}^{ \pm}$be the simple zeroes of $\operatorname{det} \boldsymbol{a}^{ \pm}(\lambda)$ and $\operatorname{det} \boldsymbol{c}^{ \pm}(\lambda)$. Then

1. $R^{ \pm}(x, y, \lambda)$ is an analytic function of $\lambda$ for $\lambda \in \mathbb{C}_{ \pm}$having pole singularities at $\lambda_{j}^{ \pm} \in \mathbb{C}_{ \pm}$;

2. $R^{ \pm}(x, y, \lambda)$ is a kernel of a bounded integral operator for $\operatorname{im} \lambda \neq 0$;

3. $R^{ \pm}(x, y, \lambda)$ is uniformly bounded function for $\lambda \in \mathbb{R}$ and provides a kernel of an unbounded integral operator;

4. $R^{ \pm}(x, y, \lambda)$ satisfy the equation

$$
L(\lambda) R^{ \pm}(x, y, \lambda)=\mathbb{1} \delta(x-y)
$$

\section{Sketch of the proof:}

1. is obvious from the fact that $\chi^{ \pm}(x, \lambda)$ are the FAS of $L(\lambda)$. From definition (44) it follows $\chi^{ \pm}(x, \lambda)$ and $\hat{\chi}^{ \pm}(y, \lambda)$, and consequently, $R^{ \pm}(x, y, \lambda)$ will develop pole singularities for all $\lambda_{j}^{ \pm}$for which $\operatorname{det} \boldsymbol{a}^{ \pm}(\lambda)=0$.

2. Assume that $\operatorname{im} \lambda>0$ and consider the asymptotic behavior of $R^{+}(x, y, \lambda)$ for $x, y \rightarrow \infty$. From equations (44), (45) we find that

$$
R^{+}(x, y, \lambda)=\sum_{p=1}^{n} X^{+}(x, \lambda) \mathrm{e}^{-\mathrm{i} \lambda J(x-y)} \Theta^{+}(x-y) \hat{X}^{+}(y, \lambda) .
$$

Due to the fact that $\chi^{+}(x, \lambda)$ has block-triangular asymptotics for $x \rightarrow \infty$ and $\lambda \in \mathbb{C}_{+}$and for the correct choice of $\Theta^{+}(x-y)$ (76) we check that the right hand side of (78) falls off exponentially for $x \rightarrow \infty$ and arbitrary choice of $y$. All other possibilities are treated analogously.

3. For $\lambda \in \mathbb{R}$ the arguments of item 2) can not be applied because the exponentials in the right hand side of (78) for $\operatorname{im} \lambda=0$ only oscillate. Thus we conclude that $R^{ \pm}(x, y, \lambda)$ for $\lambda \in \mathbb{R}$ is only a bounded function for $x \rightarrow \pm \infty$ and thus the corresponding operator $R(\lambda)$ is an unbounded integral operator.

4. The proof of equation (77) follows from the fact that $L(\lambda) \chi^{+}(x, \lambda)=0$ and

$$
\frac{\mathrm{d} \Theta^{ \pm}(x-y)}{\mathrm{d} x}=\mathbb{1} \delta(x-y)
$$


which concludes the proof.

From Theorem 6, item 3) there follows that the continuous spectrum of $L$ fills up the whole real $\lambda$-axis with multiplicity $n$. By definition the operator $L$ may also have discrete eigenvalues at the points at which $R^{ \pm}(x, y, \lambda)$ have pole singularities. From item 1) it follows that these are precisely the points $\lambda_{j}^{ \pm}$.

Let us now outline the structure of these singularities and evaluate the corresponding residues. The result is that $R^{ \pm}(x, y, \lambda)$ have poles of first order in the neighborhood of $\lambda_{j}^{ \pm}$with residues [12]

$$
\operatorname{Res}_{\lambda=\lambda_{j}^{ \pm}} R^{ \pm}(x, y, \lambda)= \pm \mathrm{i}\left|\psi_{j}^{ \pm}(x)\right\rangle \rho_{j}^{ \pm}\left\langle\psi_{j}^{ \pm}(y)\right|
$$

where $\rho_{j}^{ \pm}$are defined in equation (70).

Now we can derive the completeness relation for the eigenfunctions of the Lax operator by applying the contour integration method (see, e.g., $[1,18]$ ) to the integral

$$
\mathcal{J}(x, y)=\frac{1}{2 \pi \mathrm{i}} \oint_{\gamma_{+}} \mathrm{d} \lambda R^{+}(x, y, \lambda)-\frac{1}{2 \pi \mathrm{i}} \oint_{\gamma_{-}} \mathrm{d} \lambda R^{-}(x, y, \lambda)
$$

where the contours $\gamma_{ \pm}$are shown in Figure 1. Skipping the details we get

$$
\begin{aligned}
& \delta(x-y) J \\
& =\frac{1}{2 \pi} \int_{-\infty}^{\infty} \mathrm{d} \lambda\left\{\left|\phi^{+}(x, \lambda)\right\rangle \hat{\boldsymbol{a}}^{+}(\lambda)\left\langle\hat{\psi}^{+}(y, \lambda)|-| \phi^{-}(x, \lambda)\right\rangle \hat{\boldsymbol{a}}^{-}(\lambda)\left\langle\hat{\psi}^{-}(y, \lambda)\right|\right\} \\
& \quad-\mathrm{i} \sum_{j=1}^{N}\left(\left|\psi_{j}^{+}(x)\right\rangle \rho_{j}^{+}\left\langle\psi_{j}^{+}(y)|-| \psi_{j}^{-}(x)\right\rangle \rho_{j}^{-}\left\langle\psi_{j}^{-}(y)\right|\right) .
\end{aligned}
$$

The completeness relation (82) is a natural generalization of the one in [18] for the $\mathfrak{s l}(2)$ case. An important difference here is that now we have matrix-valued spectral functions $\boldsymbol{a}^{ \pm}(\lambda)$ whose zeroes determine the location of the discrete eigenvalues. It also allows us to introduce the minimal sets of scattering data.

Lemma 7. Let the potential $q(x)$ is such that the Lax operator $L$ satisfies the involution (66a) and has a finite number of discrete eigenvalues. Then as minimal set of scattering data which determines uniquely the scattering matrix $T(\lambda)$ and the corresponding potential $q(x)$ one can consider either one of the sets $\mathfrak{T}_{i}, i=$ 1,2

$$
\begin{aligned}
& \mathfrak{T}_{1} \equiv\left\{\tau_{\alpha}^{+}(\lambda), \quad \alpha \in \Delta_{1}^{+} \quad \lambda \in \mathbb{R} ; \quad \lambda_{k}^{+}, \quad \tau_{k}^{+}, \quad k=1, \ldots, N\right\} \\
& \mathfrak{T}_{2} \equiv\left\{\rho^{+}(\lambda), \quad \alpha \in \Delta_{1}^{+} \quad \lambda \in \mathbb{R} ; \quad \lambda_{k}^{+}, \quad \rho_{k}^{+}, \quad k=1, \ldots, N\right\} .
\end{aligned}
$$




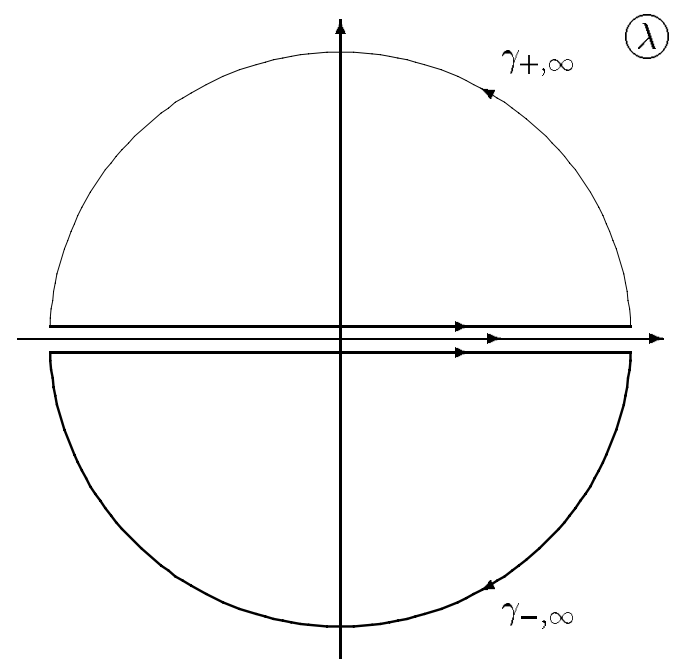

Figure 1. The contours $\gamma_{ \pm}=\mathbb{R} \cup \gamma_{ \pm \infty}$.

Outline of the proof: First we consider the class of potentials for which the Lax operator has no discrete eigenvalues, i.e., $N=0$.

Let $\mathfrak{T}_{1}$ be given. Using the involution (68) we determine also $\tau^{-}(\lambda)$. Thus we easily construct $S^{ \pm}(\lambda)$ and the sewing functions $G_{0, J}(\lambda)$ and $G_{J}(x, \lambda)$, see equation (53). The next step consists in solving the RHP with canonical normalization for the FAS $X^{ \pm}(x, \lambda)$. Since the Lax operator has no discrete eigenvalues, $X^{ \pm}(x, \lambda)$ are regular solutions of the RHP and therefore are uniquely determined.

The next step is to use the asymptotics of $X^{ \pm}(x, \lambda)$ for $x \rightarrow \pm \infty$. From equations (44), (50) we have

$$
\lim _{x \rightarrow \pm \infty} X^{ \pm}(x, \lambda)=T^{\mp} D^{ \pm}(\lambda)
$$

whose block diagonal part gives us both $D^{+}(\lambda)$ and $D^{-}(\lambda)$. Finally from the right hand sides of (84) we recover uniquely also $T^{\mp}(\lambda)$ and $\mathfrak{T}_{2}$. The reconstruction of $T(\lambda)$ is easily done, since we know its Gauss factors.

Let us explain how, given the solutions $X^{ \pm}(x, \lambda)$ one recovers the corresponding potential $q(x)$. Since $X^{ \pm}(x, \lambda)$ are solution of a RHP with canonical normalization they allow asymptotic expansions for $\lambda \rightarrow \infty$

$$
X^{ \pm}(x, \lambda)=\mathbb{1}+\sum_{k=1}^{\infty} \lambda^{-k} X_{k}^{ \pm}(x), \quad \hat{X}^{ \pm}(x, \lambda)=\mathbb{1}+\sum_{k=1}^{\infty} \lambda^{-k} \hat{X}_{k}^{ \pm}(x) .
$$


It remains to remember that $\chi^{ \pm}(x, \lambda)=X^{ \pm}(x, t, \lambda) \mathrm{e}^{\mathrm{i} \lambda J x}$ is a fundamental solution of $L$. Inserting it into equation (2) and taking the limit $\lambda \rightarrow \infty$ we get

$$
q(x)=\lim _{\lambda \rightarrow \infty} \lambda\left(J-X^{ \pm} J \widehat{X}^{ \pm}(x, \lambda)\right)=\left[J, X_{1}^{ \pm}(x)\right]
$$

where we took into account that $X_{1}^{ \pm}(x)=-\hat{X}_{1}^{ \pm}(x)$.

The reconstruction of $T(\lambda)$ and $q(x)$ from $\mathfrak{T}_{2}$ can be done in an analogous way using the FAS

$$
X^{\prime, \pm}(x, \lambda) \equiv X^{ \pm}(x, \lambda) \hat{D}^{ \pm}=\psi^{ \pm}(x, \lambda) T^{\mp}(\lambda)=\phi^{ \pm}(x, \lambda) S^{ \pm}(\lambda) \hat{D}^{ \pm}(\lambda) .
$$

If $N>1$ the FAS are solutions of singular RHP. In this case we have to give additional considerations to show that $\rho_{k}^{+}$and $\lambda_{k}^{+}$fix up uniquely the corresponding singular solutions of the RHP. Such considerations are based on the dressing Zakharov-Shabat method [32].

We finish this Section by noting, that using the FAS one can construct the "squared solutions" of $L$ which map the potential $q(x)$ to the corresponding sets of minimal scattering data $\mathfrak{T}_{i}, i=1,2$. Then one can prove the completeness relation for the "squared solutions" and interpret the mapping $q(x) \rightarrow \mathfrak{T}_{i}$ as a generalized Fourier transform. In fact the completeness relation of the "squared solutions" provides the spectral decomposition for the operators $\Lambda_{ \pm}$that generate the corresponding class of NLEE.

\section{Spectral Properties of Lax Operators on Symmetric Spaces. Constant Boundary Conditions}

Here we show that the constant boundary conditions substantially modify the form of the spectrum of $L$ and the construction of the FAS. Nevertheless our aim is to demonstrate that FAS can be constructed also in this case and the programm outlined above can be implemented also for soliton equations with CBC.

We start by defining the notion of an admissible potential $q(x)$ satisfying the above conditions and ensuring regular solutions to the direct and inverse scattering problems for $L$. First we note that the manifold $\mathcal{M}$ of all admissible potentials is a nonlinear one, i.e., linear combination of two admissible potentials generically is not admissible. 


\subsection{Jost Solutions and Continuous Spectrum of $L$}

Like for the case of VBC, the Jost solutions of $L$ with CBC are determined by their asymptotics for $x \rightarrow \pm \infty$. This is the crucial point which is the source of the substantial differences between the two cases. In order to determine these asymptotics we need to find the eigenfunctions of the two asymptotic operators

$$
\begin{gathered}
L_{+} \psi_{\text {as }}(x, \lambda) \equiv \mathrm{i} \frac{\mathrm{d} \psi_{\mathrm{as}}}{\mathrm{d} x}+q_{+} \psi_{\mathrm{as}}(x, \lambda)-\lambda J \psi_{\mathrm{as}}(x, \lambda)=0 \\
L_{-} \phi_{\mathrm{as}}(x, \lambda) \equiv \mathrm{i} \frac{\mathrm{d} \phi_{\mathrm{as}}}{\mathrm{d} x}+q_{-} \phi_{\mathrm{as}}(x, \lambda)-\lambda J \phi_{\mathrm{as}}(x, \lambda)=0 \\
q_{ \pm}=\lim _{x \rightarrow \pm \infty} q(x)=\left(\begin{array}{cc}
0 & \boldsymbol{q}_{ \pm}^{T}(x, t) \\
-\boldsymbol{q}_{ \pm}^{*}(x, t) & 0
\end{array}\right), \quad J=\left(\begin{array}{cc}
1 & 0 \\
0 & -\mathbb{1}
\end{array}\right) .
\end{gathered}
$$

Here we already imposed on the Lax operator the involution (66b), see also Remark 5 .

The two asymptotic operators $L_{ \pm}$are ordinary differential operators with constant coefficients. In order to calculate their fundamental solutions we need to diagonalize the matrices $U_{ \pm} \equiv q_{ \pm}-\lambda J$ and find their eigenvalues. The answer is as follows

$$
U_{+}(\lambda) \psi_{0}(\lambda)=-\psi_{0}(\lambda) J(\lambda), \quad U_{-}(\lambda) \phi_{0}(\lambda)=-\phi_{0}(\lambda) J(\lambda)
$$

where the eigenvalues are

$$
\pm j_{k}(\lambda)= \pm \sqrt{\lambda^{2}-\rho_{k}^{2}}, \quad k=1, \ldots, s
$$

and an eigenvalue $\lambda$ with multiplicity $s^{\prime}-s$. We will arrange them in a diagonal matrix $J(\lambda)$ as follows

$$
J(\lambda)=\operatorname{diag}(j_{1}(\lambda), \ldots, j_{s}(\lambda), \underbrace{\lambda, \ldots, \lambda}_{s^{\prime}-s},-j_{s}(\lambda), \ldots,-j_{1}(\lambda)) .
$$

The matrices $\psi_{0}(\lambda)$ and $\phi_{0}(\lambda)$ are of the form

$$
\begin{array}{rlrl}
\psi_{0}(\lambda) & =\varphi_{0}^{+} U_{0}(\lambda), & \phi_{0}(\lambda) & =\varphi_{0}^{-} U_{0}(\lambda) \\
\varphi_{0}^{ \pm} & =\left(\begin{array}{cc}
\underline{\varphi}_{1}^{ \pm} & 0 \\
0 & \underline{\varphi}_{2}^{ \pm}
\end{array}\right), & U_{0}(\lambda)=\left(\begin{array}{ccc}
\underline{A} & 0 & \underline{B} \\
0 & \mathbb{1}_{s^{\prime}-s} & 0 \\
\underline{B} & 0 & \underline{A}
\end{array}\right) .
\end{array}
$$


Here the $s \times s$ matrix $\underline{\varphi}_{1}^{ \pm}$and $s^{\prime} \times s^{\prime}$ matrix $\underline{\varphi}_{2}^{ \pm}$are determined by

$$
\begin{array}{ll}
\boldsymbol{q}_{ \pm} \boldsymbol{q}_{ \pm}^{\dagger} \underline{\varphi}_{1}^{ \pm}=\underline{\varphi}_{1}^{ \pm} \underline{\rho}, & \boldsymbol{q}_{ \pm}^{\dagger} \boldsymbol{q}_{ \pm} \underline{\varphi}_{2}^{ \pm}=\underline{\varphi}_{2}^{ \pm} \underline{\rho}^{\prime} \\
\underline{A}_{j k}=A_{k} \delta_{j, k}, & \underline{B}_{j k}=B_{k} \delta_{j, s+1-k} \\
A_{k}=\sqrt{\frac{\lambda+j_{k}}{2 j_{k}},} & B_{k}=\sqrt{\frac{\lambda-j_{k}}{2 j_{k}}} \\
\underline{\rho}=\operatorname{diag}\left(\rho_{1}^{2}, \ldots, \rho_{s}^{2}\right), & \underline{\rho}^{\prime}=\operatorname{diag}(\underbrace{0, \ldots, 0}_{s^{\prime}-s}, \rho_{s}^{2}, \ldots, \rho_{1}^{2}) .
\end{array}
$$

Remark 8. Without loss of generality we can consider $\psi_{0}(\lambda)$ and $\phi_{0}(\lambda)$ as group elements. In the case of A.III symmetric spaces we assume that $\varphi_{1}^{ \pm}$and $\varphi_{2}^{ \pm}$are elements of the groups $\mathrm{SU}(s)$ and $\mathrm{SU}\left(s^{\prime}\right)$, respectively. Similarly, for C.II-type (respectively D.III-type) symmetric spaces we will choose $n=2\left(s+s^{\prime}\right)$ and $\underline{\varphi}_{1}^{ \pm} \in \mathrm{Sp}(2 s), \underline{\varphi}_{2}^{ \pm} \in \mathrm{Sp}\left(2 s^{\prime}\right)$ (respectively $\underline{\varphi}_{1}^{ \pm} \in \mathrm{SO}(2 s), \underline{\varphi}_{2}^{ \pm} \in \mathrm{SO}\left(2 s^{\prime}\right)$ ).

Remark 9. For reasons, that will become clear below, we require that the two asymptotic operators have the same spectrum, i.e., we imposed the condition $q_{+}^{2}=$ $q_{-}^{2}$, which in block components gives $\boldsymbol{q}_{+}^{\dagger} \boldsymbol{q}_{+}=\boldsymbol{q}_{-}^{\dagger} \boldsymbol{q}_{-}$and $\boldsymbol{q}_{+} \boldsymbol{q}_{+}^{\dagger}=\boldsymbol{q}_{-} \boldsymbol{q}_{-}^{\dagger}$.

More specifically, we assume that there exist a block diagonal matrix $W_{0}$ which is an integral of motion for the MNLSE which commutes with $J(\lambda)$, i.e.,

$$
\begin{gathered}
q_{-}=W_{0}^{-1} q_{+} W_{0}, \quad W_{0}=\left(\begin{array}{ccc}
w_{01} & 0 & 0 \\
0 & w_{02} & 0 \\
0 & 0 & s_{0} w_{01} \hat{s}_{0}
\end{array}\right) \\
w_{01}=\operatorname{diag}\left(w_{01 ; 1}, \ldots, w_{01 ; s}\right), \quad w_{02} \in \mathrm{SU}\left(s^{\prime}-s\right) .
\end{gathered}
$$

Generalizing the ideas, developed by Faddeev and Takhtadjan in [28] we split the manifold $\mathcal{M}$ into the union of disjoint submanifolds [17]

$$
\mathcal{M}=\bigcup_{W_{0}, q_{+}} \mathcal{M}_{W_{0}, q_{+}}
$$

on each of which besides the limiting value $q_{+}$also the integral of motion $W_{0}$ is fixed up. More precisely, the elements of $\mathcal{M}_{W_{0}, q_{+}}$are the matrix-valued functions $q(x)$ of the form as in (2) and satisfying (93).

The operators $L_{ \pm}$have purely continuous spectrum, determined by the eigenvalues $\rho_{k}^{2}$ of the matrices $\psi_{0}(\lambda)$ and $\phi_{0}(\lambda)$, which due to the involution, must be real. 
For definiteness we will choose them to be all different

$$
\rho_{1}>\rho_{2}>\cdots>\rho_{s}>0 .
$$

Cases when subsets of $\left\{\rho_{k}\right\}$ are equal can be considered analogously.

Obviously, each of the eigenvalues $j_{k}(\lambda)$ in fact introduces a Riemannian surface $\mathfrak{R}_{k}$ which has two leafs $\mathfrak{R}_{k}=\mathfrak{R}_{k}^{+} \cup \mathfrak{R}_{k}^{-}$corresponding to the sign of $\operatorname{im} j_{k}(\lambda)$. So in fact we have to deal with the collection of Riemannian surfaces $\mathfrak{R}$

$$
\mathfrak{R}=\mathbb{C} \cup \bigcup_{k=1}^{s} \mathfrak{R}_{k}
$$

With all this the solutions of equation (88) take the form

$$
\psi_{\text {as }}(x, \lambda)=\psi_{0}(\lambda) \mathrm{e}^{-\mathrm{i} J(\lambda) x}, \quad \phi_{\text {as }}(x, \lambda)=\phi_{0}(\lambda) \mathrm{e}^{-\mathrm{i} J(\lambda) x} .
$$

Note that the exponential factors in the right hand side of equation (97) become oscillating for different intervals on the real $\lambda$-axis. Indeed, $\exp \left( \pm \mathrm{i} j_{k}(\lambda) x\right)$ oscillates on two semi-intervals on the real $\lambda$-axis

$$
\ell_{k}^{-} \equiv\left(-\infty<\operatorname{Re} \lambda \leq-\rho_{k}\right], \quad \ell_{k}^{+} \equiv\left[\rho_{k} \leq \operatorname{Re} \lambda<\infty\right)
$$

In fact these are the cuts that determine the Riemannian surface $\mathfrak{R}_{k}$. So it is natural to introduce the projectors

$$
P_{k}(\lambda)=\theta\left(|\operatorname{Re} \lambda|-\rho_{k}\right), \quad k=1, \ldots, s
$$

which, when applied to the real axis, pick up $\ell_{k}=\ell_{k}^{-} \cup \ell_{k}^{+}$.

Now we are ready to state that the continuous spectrum of $L_{ \pm}$consists of the union $\cup_{k=1}^{s} \ell_{s} \cup \mathbb{R}$. The multiplicity of the spectrum on each $\ell_{k}$ is two.

The continuous spectrum of $L$ coincides with the spectra of $L_{ \pm}$, see Figure 2.

\subsection{Jost Solutions, Scattering Matrix and FAS}

We introduce the Jost solutions as follows

$$
\begin{gathered}
\lim _{x \rightarrow \infty} \psi(x, \lambda) \mathrm{e}^{\mathrm{i} J(\lambda) x}=\psi_{0}(\lambda) P(\lambda) \\
\lim _{x \rightarrow-\infty} \phi(x, \lambda) \mathrm{e}^{\mathrm{i} J(\lambda) x}=\phi_{0}(\lambda) P(\lambda) \\
P(\lambda)=\sum_{k=1}^{s} P_{k}(\lambda)\left(E_{k, k}+E_{\bar{k}, \bar{k}}\right)+P_{0}, \quad P_{0}=\sum_{k=s+1}^{s^{\prime}} E_{k, k}
\end{gathered}
$$




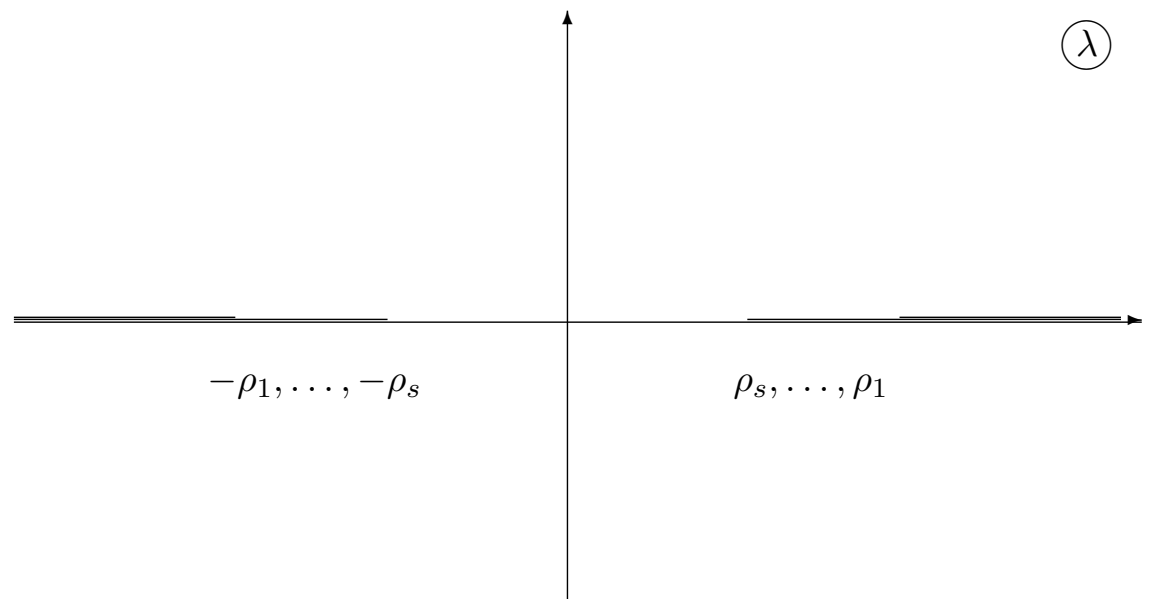

Figure 2. The continuous spectrum of the operators $L_{ \pm}$and $L$.

with $\bar{k}=n+1-k$.

The presence of the projector $P(\lambda)$ in the definition of the Jost solutions reflects the first important difference between the VBC and CBC case: it reflects the fact that the multiplicity of the continuous spectrum of $L$ is no more constant. It means also that the rank of the Jost solution varies with $\lambda \in \mathbb{R}$

$$
\begin{array}{lll}
\operatorname{rank} \psi(x, \lambda)=\operatorname{rank} \phi(x, \lambda)=n & \text { for } & |\operatorname{Re} \lambda| \geq \rho_{1} \\
\operatorname{rank} \psi(x, \lambda)=\operatorname{rank} \phi(x, \lambda)=n-2 k & \text { for } & \rho_{k}>|\operatorname{Re} \lambda| \geq \rho_{k+1} \\
\operatorname{rank} \psi(x, \lambda)=\operatorname{rank} \phi(x, \lambda)=s^{\prime}-s & \text { for } & \rho_{s}>|\operatorname{Re} \lambda| .
\end{array}
$$

This means also that the Jost solution does not have inverse for any $\lambda$. Therefore, we slightly modify the definitions of $\hat{\psi}(x, \lambda)$ and $\hat{\phi}(x, \lambda)$ so that they exist on the image of $P(\lambda)$. We consider them as the Jost solutions of the dual linear problem

$$
\mathrm{i} \frac{\mathrm{d} \hat{\psi}}{\mathrm{d} x}-\hat{\psi}(x, \lambda)(q(x)-\lambda J)=0 .
$$

This linear problem also has Jost solutions which are introduced by

$$
\begin{aligned}
\lim _{x \rightarrow \infty} \mathrm{e}^{-\mathrm{i} J(\lambda) x} \psi(x, \lambda) & =P(\lambda) \hat{\psi}_{0}(\lambda) \\
\lim _{x \rightarrow-\infty} \mathrm{e}^{-\mathrm{i} J(\lambda) x} \phi(x, \lambda) & =P(\lambda) \hat{\phi}_{0}(\lambda) \\
\hat{\psi}_{0}(\lambda)=\hat{\varphi}_{0}^{+} \hat{U}_{0}(\lambda), \quad \hat{\phi}_{0}(\lambda) & =\hat{\varphi}_{0}^{-} \hat{U}_{0}(\lambda) .
\end{aligned}
$$


Note also that $\psi_{0}(\lambda)$ and $\phi_{0}(\lambda)$ and their inverse depend on $\lambda$ only through $U_{0}(\lambda)$ and that

$$
\hat{U}_{0}(\lambda)=\left(\begin{array}{ccc}
\underline{A} & 0 & -\underline{B}^{T} \\
0 & \mathbb{1}_{s /-s} & 0 \\
-\underline{B}^{T} & 0 & \underline{A}
\end{array}\right) .
$$

In proving that $\hat{U}_{0}(\lambda) U_{0}(\lambda)=\mathbb{1}$ we use the relations

$$
A_{k}^{2}-B_{k}^{2}=1, \quad k=1, \ldots, s .
$$

Next we derive the integral equations for the Jost solutions. To this end it is convenient to introduce the quantities

$$
X(x, \lambda)=\hat{\psi}_{0} \psi(x, \lambda) \mathrm{e}^{\mathrm{i} J(\lambda) x}, \quad Y(x, \lambda)=\hat{\phi}_{0} \phi(x, \lambda) \mathrm{e}^{\mathrm{i} J(\lambda) x}
$$

which satisfy the equations

$$
\begin{aligned}
& \mathrm{i} \frac{\mathrm{d} X}{\mathrm{~d} x}+Q_{+}(x, \lambda) X(x, \lambda)-[J(\lambda), X(x, \lambda)]=0 \\
& \mathrm{i} \frac{\mathrm{d} Y}{\mathrm{~d} x}+Q_{-}(x, \lambda) Y(x, \lambda)-[J(\lambda), Y(x, \lambda)]=0
\end{aligned}
$$

where the "potentials" $Q_{ \pm}(x, \lambda)$

$$
Q_{+}(x, \lambda)=\hat{\psi}_{0}\left(q(x)-q_{+}\right) \psi_{0}(\lambda), \quad Q_{-}(x, \lambda)=\hat{\phi}_{0}\left(q(x)-q_{-}\right) \phi_{0}(\lambda)
$$

now depend also on the spectral parameter. Since from equation (100) there follows that

$$
\lim _{x \rightarrow \infty} X(x, \lambda)=\mathbb{1}, \quad \lim _{x \rightarrow-\infty} Y(x, \lambda)=\mathbb{1}
$$

we are able to derive the following integral equations for $X(x, \lambda)$ and $Y(x, \lambda)$

$$
\begin{aligned}
& X(x, \lambda)=\mathbb{1}+\mathrm{i} \int_{\infty}^{x} \mathrm{~d} y \mathrm{e}^{-\mathrm{i} J(\lambda)(x-y)} Q_{+}(x, \lambda) X(x, \lambda) \mathrm{e}^{\mathrm{i} J(\lambda)(x-y)} \\
& Y(x, \lambda)=\mathbb{1}+\mathrm{i} \int_{-\infty}^{x} \mathrm{~d} y \mathrm{e}^{-\mathrm{i} J(\lambda)(x-y)} Q_{-}(x, \lambda) Y(x, \lambda) \mathrm{e}^{\mathrm{i} J(\lambda)(x-y)} .
\end{aligned}
$$

The scattering matrix is introduced like in the VBC case

$$
T(\lambda)=\hat{\psi}(x, \lambda) \phi(x, \lambda) .
$$

The important difference as compare to the VBC case concerns the analyticity properties of the different columns of the Jost solutions. For VBC we verified 
(see equation (33)) that each of the columns of the Jost solutions can be extended analytically either for $\lambda \in \mathbb{C}_{+}$or for $\lambda \in \mathbb{C}_{-}$.

For CBC the picture is substantially different. First, now we are dealing with analyticity on the sheets of the Riemannian surface described above. The second difference is in the fact, that now $J(\lambda)$ has more than two different eigenvalues. From this point of view the problem of constructing the FAS for CBC resembles the one for $N$-wave problem [27,30]. The first step of the solution is to introduce an ordering in the matrix elements of $J(\lambda)$. From equation (95) we find, that an ordering $\operatorname{im} J_{k}(\lambda)>\operatorname{im} J_{l}(\lambda)$ for $k<l$ can be introduced on the main leaf $\mathfrak{R}^{+}=\mathbb{C}_{+} \cup \cup_{k=1}^{s} \mathfrak{R}_{k}^{+}$of the Riemannian surface. Indeed, one can check that from equation (95) there follows

$$
\operatorname{im} j_{1}(\lambda)>\operatorname{im} j_{2}(\lambda)>\cdots>\operatorname{im} j_{s}(\lambda)>0, \quad \lambda \in \mathfrak{R}^{+} .
$$

Using this ordering we will outline how it is possible to construct a FAS on the main leaf $\mathfrak{R}^{+}$and on its antipode leaf $\mathfrak{R}^{-}=\mathbb{C}_{-} \cup \cup_{k=1}^{s} \mathfrak{R}_{k}^{-}$. Similar procedure exists for each of the other leafs of the Riemannian surface. In order to simplify the analysis we assume that $s^{\prime}=s$.

Skipping the details we formulate the procedure of constructing the FAS $Z^{ \pm}(x, \lambda)$ on the leafs $\mathcal{R}^{ \pm}$. Since any two fundamental solutions must be linearly related we first introduce them by

$$
Z^{ \pm}(x, \lambda)=\phi(x, \lambda) S^{ \pm}(\lambda)=\psi(x, \lambda) T^{\mp}(\lambda) D^{\mp}(\lambda)
$$

where $S^{ \pm}(\lambda), T^{ \pm}(\lambda)$ and $D^{ \pm}(\lambda)$ are the factors of the Gauss decomposition [21] of the scattering matrix

$$
T(\lambda)=T^{-}(\lambda) D^{+}(\lambda) \hat{S}^{+}(\lambda) .
$$

In other words, $T^{+}$and $S^{+}$(respectively $T^{-}$and $S^{-}$) are upper- (respectively lower-) triangular matrices taking values in the corresponding group and $D^{ \pm}(\lambda)$ are diagonal matrices (i.e., elements of the Cartan subgroup).

The proof of the fact that $Z^{+}(x, \lambda)$ (respectively $Z^{-}(x, \lambda)$ ) is analytic for $\lambda \in \mathfrak{R}^{+}$ (respectively for $\lambda \in \mathfrak{R}^{-}$) is based on the analysis of the set of integral equations that $Z^{ \pm}(x, \lambda)$ satisfy. It is more convenient to write down those equations for $\tilde{Z}^{ \pm}(x, \lambda)=\hat{\phi}_{0}(\lambda) Z^{ \pm}(x, \lambda) \mathrm{e}^{\mathrm{i} J(\lambda) x}$

$$
\tilde{Z}_{k p}^{+}(x, \lambda)=\delta_{k p}+\mathrm{i} \int_{-\infty}^{x} \mathrm{~d} y \mathrm{e}^{-\mathrm{i}\left(J_{k}(\lambda)-J_{p}(\lambda)\right)(x-y)} \sum_{a=1}^{n} Q_{-; k a}(y, \lambda) \tilde{Z}_{a p}^{+}(y, \lambda)
$$


for $k \geq p$ and

$$
\tilde{Z}_{k p}^{+}(x, \lambda)=\mathrm{i} \int_{\infty}^{x} \mathrm{~d} y \mathrm{e}^{-\mathrm{i}\left(J_{k}(\lambda)-J_{p}(\lambda)\right)(x-y)} \sum_{a=1}^{n} Q_{-; k a}(y, \lambda) \tilde{Z}_{a p}^{+}(y, \lambda)
$$

for $k<p$ and $\lambda \in \mathfrak{R}^{+}$. Similarly we have

$$
\tilde{Z}_{k p}^{-}(x, \lambda)=\delta_{k p}+\mathrm{i} \int_{-\infty}^{x} \mathrm{~d} y \mathrm{e}^{-\mathrm{i}\left(J_{k}(\lambda)-J_{p}(\lambda)\right)(x-y)} \sum_{a=1}^{n} Q_{-; k a}(y, \lambda) \tilde{Z}_{a p}^{-}(y, \lambda)
$$

for $k \leq p$ and

$$
\tilde{Z}_{k p}^{-}(x, \lambda)=\mathrm{i} \int_{-\infty}^{x} \mathrm{~d} y \mathrm{e}^{-\mathrm{i}\left(J_{k}(\lambda)-J_{p}(\lambda)\right)(x-y)} \sum_{a=1}^{n} Q_{-; k a}(y, \lambda) \tilde{Z}_{a p}^{-}(y, \lambda)
$$

for $k>p$ and $\lambda \in \mathfrak{R}^{-}$.

The analyticity properties follow from the fact that with the above ordering of the $\operatorname{im} j_{k}(\lambda)$ all exponential factors in the integrands of (119) (respectively in the integrands of (120)) fall off for $\lambda \in \mathfrak{R}^{+}$(respectively for $\lambda \in \mathfrak{R}^{-}$).

It remains to note that the matrix elements of the Gaussian factors $T^{ \pm}(\lambda), S^{ \pm}(\lambda)$ and $D^{ \pm}(\lambda)$ can be expressed explicitly as functions of the matrix elements of $T(\lambda)$. The corresponding formulae are well known [11,21]. Here we will write down only the expressions for the matrix elements of $D^{ \pm}(\lambda)$ for the case when $T(\lambda) \in \mathrm{SL}(n)$ :

$$
D_{k p}^{+}(\lambda)=\frac{m_{k}^{+}(\lambda)}{m_{k-1}^{+}(\lambda)} \delta_{k p}, \quad D_{k p}^{-}(\lambda)=\frac{m_{n-k+1}^{-}(\lambda)}{m_{n-k}^{-}(\lambda)} \delta_{k p}
$$

where $m_{k}^{+}(\lambda)$ (respectively $m_{k}^{-}(\lambda)$ ) is the principal upper-minor (respectively principle lower-minor) of $T(\lambda)$. Here we assume that $m_{0}^{ \pm}(\lambda)=1$; recall also that $\operatorname{det} T(\lambda)=1$, i.e., $m_{n}^{ \pm}(\lambda)=1$.

Since $\tilde{Z}^{+}(x, \lambda)$ (respectively $\tilde{Z}^{-}(x, \lambda)$ ) are analytic for $\lambda \in \mathfrak{R}^{+}$(respectively for $\lambda \in \mathfrak{R}^{-}$) we find, that also their asymptotics for $x \rightarrow \infty$ are also analytic. As a result the next lemma follows.

Lemma 10. The principle minors $m_{k}^{+}(\lambda), k=1, \ldots, n-1$ (respectively $m_{k}^{-}(\lambda)$, $k=1, \ldots, n-1)$ are analytic functions of $\lambda$ for $\lambda \in \mathfrak{R}^{+}$(respectively for $\lambda \in \mathfrak{R}^{-}$). 
Remark 11. There exist FAS on each of the leafs of the Riemannian surface. Their construction is done using the same ideas. On each of these leafs we have different ordering of the $\operatorname{im} j_{k}(\lambda)$. So, the first step should be to make a permutation of the rows and columns of the matrices in $Q_{ \pm}(x, \lambda)$, and in $J(\lambda)$ so that the eigenvalues of the new matrix $J(\lambda)$ are ordered like in equation (116). The rest of the construction remains the same. At the end one should revert to the initial ordering of columns and rows.

\subsection{The Resolvent of $L$ and Minimal Set of Scattering Data}

The detailed analysis for the $\mathrm{CBC}$ in the general situation is rather involved and will be presented elsewhere. Here we just outline some of the ideas along which it should be done.

The first one is to show that the spectral problem for the Lax operator with CBC can be reduced to an RHP on the Riemannian surface $\mathfrak{R}$. We outlined above how one can construct the FAS not only on the main leaf $\mathfrak{R}^{+}$of $\mathfrak{R}$, but on any of the leafs $\mathfrak{R}_{k}$. If we denote by $\tilde{Z}_{(k)}^{ \pm}(x, \lambda)$ the FAS on the leaf $\mathfrak{R}_{k}$ then any two FAS will be linearly related on the intersection of the two leafs by

$$
\begin{aligned}
\tilde{Z}_{(k)}^{ \pm}(x, \lambda) & =\tilde{Z}_{(k)}^{ \pm}(x, \lambda) \tilde{G}_{(k, m)}(x, \lambda), \quad \lambda \in \mathfrak{R}_{k} \cap \mathfrak{R}_{m} \\
\tilde{G}_{(k, m)}(x, \lambda) & =\mathrm{e}^{-\mathrm{i} J(\lambda) x} \hat{S}_{(k)}^{+}(\lambda) S_{(m)}^{+}(\lambda) \mathrm{e}^{\mathrm{i} J(\lambda) x} .
\end{aligned}
$$

This RHP also has unique regular solution with canonical normalization. An important problem is to generalize the Zakharov-Shabat dressing method [32] and to construct explicitly the singular solutions of these RHP. For the one-component case this has been done $[18,28,29]$ and some particular multicomponent cases has also been treated, see $[2,19,24]$ but a lot still has to be done.

The FAS constructed above can be used to construct the kernel of the resolvent of $L$. On the main leaf this kernel is similar to the one in equation (75)

$$
R_{(1)}^{ \pm}(x, y, \lambda)=\frac{1}{\mathrm{i}} Z_{(1)}^{ \pm}(x, \lambda) \Theta^{+}(x-y) \hat{Z}_{(1)}^{ \pm}(y, \lambda), \quad \lambda \in \mathfrak{R}^{+}
$$

where the matrix $\Theta^{+}(x-y)$ is given by equation (76). What is important to do here is the derivation of the completeness relation for $L$ with CBC. This can be done again by using the contour integration method. There are two difficulties which must be resolved here. The first one is to evaluate explicitly the asymptotics of $R_{(1)}^{ \pm}(x, y, \lambda)$ for $\lambda \rightarrow \infty$. The second one is the evaluation of the jump of the resolvent through the cuts $\ell_{k}$. 
Finally one can construct the minimal set of scattering data. Like in the VBC case, we can consider the minimal sets of scattering data on the continuous spectrum to be

$$
\mathfrak{T}_{1} \equiv\left\{\tau_{\alpha}^{+}(\lambda) ; \alpha \in \Delta_{1}^{+}, \lambda \in \ell\right\}, \quad \mathfrak{T}_{2} \equiv\left\{\rho^{+}(\lambda) ; \alpha \in \Delta_{1}^{+}, \lambda \in \ell\right\}
$$

where $\ell=\cup_{k=1}^{s} \ell_{s} \cup \mathbb{R}$. The coefficients $\tau_{\alpha}^{ \pm}(\lambda), \rho_{\alpha}^{ \pm}(\lambda)$ are introduced by

$$
\tau_{\alpha}^{+}(\lambda)=\frac{\left\langle\hat{S}_{(1)}^{+} J S_{(1)}^{+}(\lambda), E_{ \pm \alpha}\right\rangle}{\left\langle E_{\alpha}, E_{-\alpha}\right\rangle}, \quad \rho_{\alpha}^{+}(\lambda)=\frac{\left\langle\hat{T}_{(1)}^{-} J T_{(1)}^{-}(\lambda), E_{ \pm \alpha}\right\rangle}{\left\langle E_{\alpha}, E_{-\alpha}\right\rangle} .
$$

Due to the varying multiplicity of the continuous spectrum here one has to take into account that each of the functions $\tau_{\alpha}^{+}(\lambda)$ and $\rho_{\alpha}^{+}(\lambda)$ has its own domain of definition. In addition one has to add also the data corresponding to the discrete spectrum of $L$.

Here we stop with the general theory and give some more concrete examples.

\section{Examples}

\subsection{Symmetric Spaces of A.III-Type and Vector NLSE}

We start with the symmetric spaces $\mathrm{SU}(n+1) / \mathrm{S}(\mathrm{U}(1) \otimes \mathrm{U}(n))$. The corresponding potential $q(x)$ and $J$ take the form

$$
q(x)=\left(\begin{array}{cc}
0 & \vec{q}^{T}(x) \\
-\vec{q}^{*}(x) & 0_{n}
\end{array}\right), \quad J=\left(\begin{array}{cc}
1 & 0 \\
0 & -\mathbb{1}_{n}
\end{array}\right) .
$$

So we have to set in the general case considered above $s=1$ and $s=n$; we also put $\rho_{1}=\rho$. Then formulae (90)-(92) simplify into

$$
\begin{gathered}
J(\lambda)=\operatorname{diag}(j_{1}(\lambda), \underbrace{\lambda, \ldots, \lambda}_{n-1},-j_{1}(\lambda)) \\
\varphi_{0}^{ \pm}=\left(\begin{array}{cc}
1 & 0 \\
0 & \underline{\varphi}_{2}^{ \pm}
\end{array}\right), \quad U_{0}(\lambda)=\left(\begin{array}{ccc}
A_{1} & 0 & B_{1} \\
0 & \mathbb{1}_{n-1} & 0 \\
B_{1} & 0 & A_{1}
\end{array}\right) .
\end{gathered}
$$

Since $\vec{q}$ is a vector the set of eigenvectors $\varphi_{2}^{ \pm}$of $\vec{q}^{*} \vec{q}^{* T}$ consist of $n-1$ normalized vectors orthogonal to $\vec{q}^{*}$. The last eigenvector corresponding to the only nonvanishing eigenvalue $\rho_{1}^{2}$ is proportional to $\vec{q}^{*}$. The Riemann surface has four 
leafs $\Re=\Re_{1}^{+} \cup \mathfrak{R}_{1}^{-} \cup \mathbb{C}_{+} \cup \mathbb{C}_{-}$. We will need also the uniformization variable $z=(\lambda+j(\lambda)) / \rho, 1 / z=(\lambda-j(\lambda)) / \rho$ and for $n=3$ we get

$$
Q_{ \pm}(x, z)=\frac{1}{4 \rho}\left(2 z A_{2}^{ \pm}+2 A_{1}^{ \pm}-\frac{2}{z} A_{-2}^{ \pm}+\frac{B_{1}^{ \pm}}{z+1}+\frac{B_{-1}^{ \pm}}{z-1}\right)
$$

where $B_{ \pm 1}^{ \pm}=A_{2}^{ \pm} \mp A_{1}^{ \pm}+A_{0}^{ \pm} \mp A_{-1}^{ \pm}+A_{-2}^{ \pm}$and

$$
\begin{aligned}
& A_{2}^{ \pm}=\left(\begin{array}{cccc}
0 & 0 & 0 & -a_{1}^{ \pm} \\
0 & 0 & 0 & 0 \\
0 & 0 & 0 & 0 \\
0 & 0 & 0 & 0
\end{array}\right), A_{-2}^{ \pm}=\left(\begin{array}{cccc}
0 & 0 & 0 & 0 \\
0 & 0 & 0 & 0 \\
0 & 0 & 0 & 0 \\
0 & 0 & 0 & a_{1}^{ \pm}
\end{array}\right), A_{1}^{ \pm}=\left(\begin{array}{cccc}
0 & 0 & 0 & 0 \\
0 & 0 & 0 & a_{3}^{ \pm} \\
0 & 0 & 0 & a_{2}^{ \pm} \\
0 & 0 & 0 & 0
\end{array}\right) \\
& A_{-1}^{ \pm}=\left(\begin{array}{cccc}
0 & 0 & 0 & 0 \\
-a_{3}^{ \pm} & 0 & 0 & 0 \\
-a_{2}^{ \pm} & 0 & 0 & 0 \\
0 & 0 & 0 & 0
\end{array}\right), \quad A_{0}^{ \pm}=\left(\begin{array}{cccc}
-h_{1}^{ \pm} & b_{3}^{ \pm} & b_{2}^{ \pm} & -a_{1}^{ \pm, *} \\
0 & 0 & 0 & 0 \\
0 & 0 & 0 & 0 \\
a_{1}^{ \pm, *} & -b_{3}^{ \pm} & -b_{2}^{ \pm} & h_{1}^{ \pm}
\end{array}\right) \\
& a_{1}^{ \pm}=\left(\boldsymbol{q}^{\dagger}(x) \boldsymbol{q}_{ \pm}\right)-\rho^{2}, \quad \quad a_{2,3}^{ \pm}=\frac{\boldsymbol{q}_{2,3 ; \pm}^{*}\left(\boldsymbol{q}^{\dagger}, \boldsymbol{q}_{ \pm}\right)-\boldsymbol{q}_{2,3}^{*}(x) \rho^{2}}{\boldsymbol{q}_{1, \pm} \rho} \\
& b_{2,3}^{ \pm}=\boldsymbol{q}_{1}(x) \boldsymbol{q}_{2,3 ; \pm}-\boldsymbol{q}_{2,3}(x) \boldsymbol{q}_{1, \pm}, \quad h_{1}^{ \pm}=a_{1}^{ \pm}(x)+a_{1}^{ \pm, *}(x) .
\end{aligned}
$$

Then using the uniformizing variable $z$ we rewrite the Lax operator in the form

$$
\begin{aligned}
& \mathrm{i} \frac{\mathrm{d} \tilde{\psi}}{\mathrm{d} x}+Q_{+}(x, z) \tilde{\psi}(x, z)-J(z) \tilde{\psi}(x, z)=0 \\
& \mathrm{i} \frac{\mathrm{d} \tilde{\phi}}{\mathrm{d} x}+Q_{-}(x, z) \tilde{\phi}(x, \lambda)-J(z) \tilde{\phi}(x, z)=0 \\
& J(z)=\frac{\rho}{2} \operatorname{diag}\left(z-\frac{1}{z},-z-\frac{1}{z},-z-\frac{1}{z},-z+\frac{1}{z}\right)
\end{aligned}
$$

where the new potential function has special dependence on $z$ with its coefficient functions $A_{j}^{ \pm}, B_{k}^{ \pm}$tend to zero for $x \rightarrow \pm \infty$.

Next we introduce Jost solutions and scattering matrix as

$$
\lim _{x \rightarrow \infty} \tilde{\psi}(x, z) \mathrm{e}^{\mathrm{i} J(z) x}=\mathbb{1}, \quad \lim _{x \rightarrow-\infty} \tilde{\phi}(x, z) \mathrm{e}^{\mathrm{i} J(z) x}=\mathbb{1}, \quad T(z)=\widehat{\tilde{\psi}} \tilde{\phi}(x, z) .
$$

The continuous spectrum of $L$ coincides with the continuous spectrum of the asymptotic operators $L_{ \pm, \text {as }}$. To this end we have to find out the curves on the 
complex $\lambda$-plane (respectively complex $z$-plane) on which $\mathrm{e}^{-\mathrm{i} J(\lambda) x}$ (respectively $\left.\mathrm{e}^{-\mathrm{i} J(z) x}\right)$ oscillates, i.e., we have to solve the equation

$$
\operatorname{im} J_{k}(z)=0, \quad k=1,2,3,4 .
$$

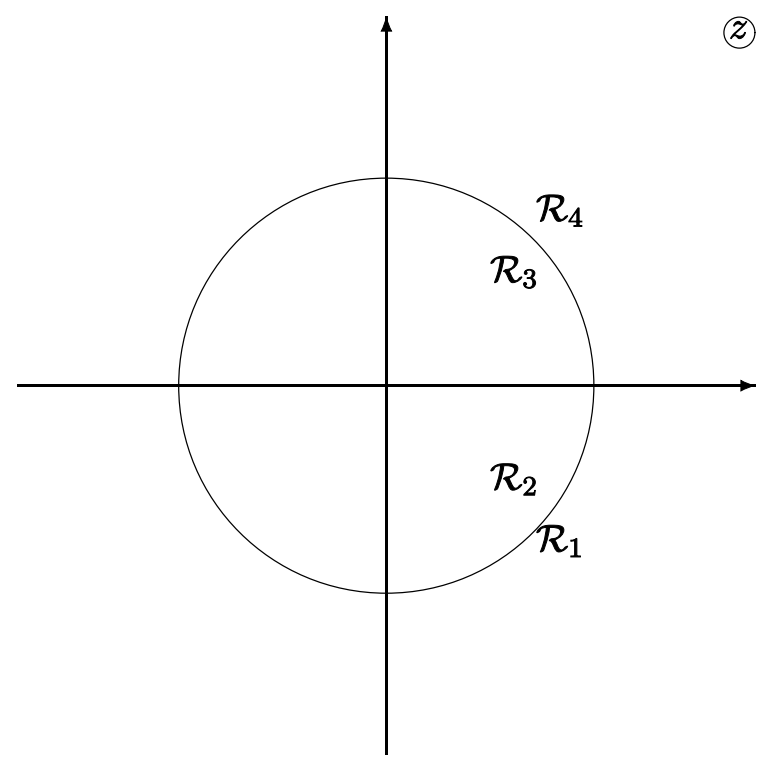

Figure 3. The continuous spectrum of $L$ on the complex $z$-plane.

In terms of the uniformizing variable fills up the real $z$-axis and the circle $S_{1}$ with radius 1 . This spectrum splits the complex $z$-plane into four regions $\mathcal{R}_{k}$, $k=1, \ldots, 4$ (see also Figure 3 )

$$
\begin{aligned}
& \mathcal{R}_{1} \equiv\{\operatorname{im} z<0, \quad|z|>1\}, \quad \mathcal{R}_{2} \equiv\{\operatorname{im} z<0, \quad|z|<1\} \\
& \mathcal{R}_{3} \equiv\{\operatorname{im} z>0, \quad|z|<1\}, \quad \mathcal{R}_{4} \equiv\{\operatorname{im} z>0, \quad|z|>1\} .
\end{aligned}
$$

In each of these regions the imaginary parts of $J_{k}(z)$ are ordered as follows

$$
\begin{aligned}
& \mathcal{R}_{1} \Rightarrow \operatorname{im} J_{1}(z)>\operatorname{im} J_{2}(z)>0>\operatorname{im} J_{4}(z) \\
& \mathcal{R}_{2} \Rightarrow \operatorname{im} J_{1}(z)>0>\operatorname{im} J_{2}(z)>\operatorname{im} J_{4}(z) \\
& \mathcal{R}_{3} \Rightarrow \operatorname{im} J_{4}(z)>\operatorname{im} J_{2}(z)>0>\operatorname{im} J_{1}(z) \\
& \mathcal{R}_{4} \Rightarrow \operatorname{im} J_{4}(z)>0>\operatorname{im} J_{2}(z)>\operatorname{im} J_{1}(z) .
\end{aligned}
$$

Remark 12. The continuous spectrum of L has variable multiplicity. Note that the conformal map from $\lambda$ to $z$ maps the semiaxis $|\operatorname{Re} \lambda|>\rho_{1}$ onto the real axis 
in the $z$-plane whereas the interval $-\rho_{1} \leq \operatorname{Re} \lambda \leq \rho_{1}$ is mapped onto the circle with radius 1 .

The construction of FAS is done again by using equation (117). The difference is that now we have to make use of a generalized Gauss decomposition (GGD) of the scattering matrix $T(\lambda)$ and its inverse $\hat{T}(\lambda)$. More specifically, let us introduce the block-matrix notations

$$
T(z)=\left(\begin{array}{lll}
T_{11} & T_{12} & T_{13} \\
T_{21} & T_{22} & T_{23} \\
T_{31} & T_{32} & T_{33}
\end{array}\right), \quad \hat{T}(z)=\left(\begin{array}{lll}
\hat{T}_{11} & \hat{T}_{12} & \hat{T}_{13} \\
\hat{T}_{21} & \hat{T}_{22} & \hat{T}_{23} \\
\hat{T}_{31} & \hat{T}_{32} & \hat{T}_{33}
\end{array}\right)
$$

where $T_{21}, T_{23}, T_{12}^{T}$, and $T_{32}^{T}$ are $n-1$-component vectors, $T_{11}, T_{13}, T_{31}, T_{33}$, are scalar functions and $T_{22}$ is an $(n-1) \times(n-1)$ matrix. Notice that this is compatible with the block-matrix structure of $J(z)$. Then the above mentioned GGD takes the form

$$
T(z)=T^{-}(z) D^{+}(z) \hat{S}^{+}(z), \quad \hat{S}^{+}(z)=\left(S^{+}\right)^{-1}(z)
$$

where $T^{-}$(respectively $S^{+}$) is lower- (respectively upper-) block-triangular matrices

$$
\begin{gathered}
T^{-}(z)=\left(\begin{array}{ccc}
1 & 0 & 0 \\
\tau_{21}^{-} & \mathbb{1} & 0 \\
\tau_{31}^{-} & \tau_{32}^{-} & 1
\end{array}\right), \\
D^{+}(z)=\operatorname{diag}\left(m_{1}^{+}, m_{2}^{+}, m_{3}^{+}\right),
\end{gathered}
$$

where

$$
\begin{array}{lll}
s_{12}^{+}=-\frac{T_{12}}{m_{1}^{+}}, & s_{23}=\hat{T}_{23} m_{3}^{+}, & s_{13}=\hat{T}_{13} m_{3}^{+} \\
\tau_{21}^{-}=-\frac{T_{21}}{m_{1}^{+}}, & \tau_{32}=\hat{T}_{32} m_{3}^{+}, & \tau_{31}=\frac{T_{31}}{m_{1}^{+}} \\
m_{1}^{+}=T_{11}, & m_{2}^{+}=T_{22}-\frac{T_{21} T_{12}}{m_{1}^{+}}, & \hat{m}_{2}^{+}=\hat{T}_{22}-\hat{T}_{21} \hat{T}_{12} m_{3}^{+} .
\end{array}
$$

As a consequence of the analyticity of $Z^{ \pm}(x, \lambda)$ we establish that $m_{1}^{+}(\lambda)$ and $m_{2}^{+}(\lambda)$ are analytic functions on $\mathfrak{R}_{1}^{+} \cap \mathbb{C}_{+}$

Remark 13. A more detailed analysis shows that the number of independent matrix elements of Gauss factors $S^{+}$and $T^{-}$is $n$, the same as the number of independent elements in the potential $q$. 
Remark 14. The Jost solutions and the scattering matrix $T(z)$ are well defined on the continuous spectrum of $L$. Thus the factors $S^{+}(z)$ and $T^{-}(z)$ in (138) are defined on the boundary of $\mathcal{R}_{1}$, whereas $\tilde{\chi}_{(1)}(x, z)$ allows analytical continuation for any $z \in \mathcal{R}_{1}$.

The construction of the FAS in any other of the sections $\mathcal{R}_{k}, k=2,3,4$ requires one additional procedure. Namely, we start first by reordering the columns and the rows of $q(x, z)-J(z)$ so that after the reordering the imaginary parts of $J(z)$ satisfy the first line of equation (136). Obviously the same reordering must be applied also to $T(z)$ and $\hat{T}(z)$. Then we apply the same GGD to the reordered $T(z)$ and $\hat{T}(z)$ and determine the corresponding $\tilde{\chi}_{(k)}(x, z)$.

The set of FAS $\tilde{Z}_{(k)}(x, z)$ satisfy the relations

$$
\tilde{Z}_{(k)}(x, z)=\tilde{Z}_{(m)}(x, z) G_{(k, m)}(x, z), \quad z \in \mathcal{R}_{k} \cap \mathcal{R}_{m}
$$

where the sewing function $G_{(k, m)}(x, z)$ is given by

$$
G_{(k, m)}(x, z)=\mathrm{e}^{\mathrm{i} J(z) x} \hat{S}_{(m)}^{+} S_{(k)}^{+} \mathrm{e}^{-\mathrm{i} J(z) x} .
$$

Imposing the condition $\lim _{z \rightarrow \infty} \tilde{\xi}_{(1)}(x, z)=\mathbb{1}$ we can treat the set of equations (141) as a generalized Riemann-Hilbert problem with canonical normalization. Thus the Inverse Scattering Problem (ISP) for the Lax operator $L$ is reduced to a RHP. We also assume that the RHP (141) has unique regular solution, i.e., solution for which $\operatorname{det} \tilde{\xi}_{(k)}(x, z) \neq 0$ for all $z \in \mathcal{R}_{k}$. Then the set of sewing functions $G_{(k, m)}(x, z)$ uniquely determines the corresponding potential $q(x, z)$ via the first asymptotic coefficients of $\tilde{\xi}_{(k)}(x, z)$ around the points $z=1, z=-1, z=0$ and $z=\infty$.

\subsection{Symmetric Spaces of C.II and D.III Type}

The first example here will be the case of $\mathfrak{g} \simeq \mathfrak{s p}(4)$ where the potential $q(x, t)$ is parametrized by three functions

$$
q(x, t)=\left(\begin{array}{cc}
0 & \boldsymbol{q}(x, t) \\
-\boldsymbol{q}^{\dagger}(x, t) & 0
\end{array}\right), \quad \boldsymbol{q}(x, t)=\left(\begin{array}{cc}
q_{12} & q_{1} \\
q_{2} & q_{12}
\end{array}\right)
$$

while the one for $\mathfrak{g} \simeq \mathfrak{s o}(8)$ contains six independent functions

$$
q(x, t)=\left(\begin{array}{cc}
0 & \boldsymbol{q}(x, t) \\
-\boldsymbol{q}^{\dagger}(x, t) & 0
\end{array}\right), \quad \boldsymbol{q}(x, t)=\left(\begin{array}{cccc}
q_{14} & q_{13} & q_{12} & 0 \\
q_{24} & q_{23} & 0 & q_{12} \\
q_{34} & 0 & q_{23} & -q_{13} \\
0 & q_{34} & -q_{24} & q_{14}
\end{array}\right) .
$$


The corresponding sets of MNLSE for these two choices of $q(x, t)$ and for VBC were first derived in [8]. For CBC with the involution (66b) they take the form

$$
\mathrm{i} \frac{\partial \boldsymbol{q}}{\partial t}+\frac{\partial^{2} \boldsymbol{q}}{\partial x^{2}}-2\left(\boldsymbol{q}^{\dagger}, \boldsymbol{q}\right) \boldsymbol{q}(x, t) \boldsymbol{q}(x, t)+\left(\boldsymbol{q}_{ \pm}^{\dagger}, \boldsymbol{q}_{ \pm}\right) \boldsymbol{q}(x, t)+\left(\boldsymbol{q}_{ \pm}^{\dagger}, \boldsymbol{q}(x, t)\right) \boldsymbol{q}_{ \pm}=0 .
$$

The additional linear in $\boldsymbol{q}$ terms ensure regular behavior for $t \rightarrow \pm \infty$ [19].

Here we briefly describe the spectrum of the corresponding Lax operators. To this end we start by determining the corresponding eigenvalues of the asymptotic matrices $q_{ \pm}$.

These eigenvalues for $q(x) \in \mathfrak{s p}(4)$ are the roots of the characteristic equation (see equation (92))

$$
\underline{\rho}^{2}-K_{0} \underline{\rho}+K_{1}=0, \quad K_{0}=\operatorname{tr} \boldsymbol{q}_{ \pm} \boldsymbol{q}_{ \pm}^{\dagger}, \quad K_{1}=\operatorname{det} \boldsymbol{q}_{ \pm} \boldsymbol{q}_{ \pm}^{\dagger}
$$

and determine the end points of the spectrum. Taking $\boldsymbol{q}_{ \pm}$to be $2 \times 2$ constant matrices as in (143) we get

$$
K_{0}=\left|q_{1 ; \pm}\right|^{2}+\left|q_{2 ; \pm}\right|^{2}+2\left|q_{12 ; \pm}\right|^{2}, \quad K_{1}=\left|\left(q_{12 ; \pm}\right)^{2}-q_{1 ; \pm} q_{2 ; \pm}\right|^{2} .
$$

Since $K_{0}>0$ and $K_{1}>0$ both roots or equation (146) must be positive. Thus we have two possibilities for the roots $\rho_{1}^{2}, \rho_{2}^{2}$ of equation (146):

a) $\rho_{1}^{2}>\rho_{2}^{2}$, i.e., the branch points are different and positive. The continuous spectrum of $L$ fills up two pairs of rays on the real axis $|\operatorname{Re} \lambda|>\rho_{1}$ and $|\operatorname{Re} \lambda|>\operatorname{Re} \rho_{2}$;

b) $\rho_{1}^{2}=\rho_{2}^{2}$, i.e., the branch points now coincide; the total multiplicity of the spectrum is 4 .

One can expect that the corresponding characteristic polynomial for $\mathfrak{s o}(8)$ is of fourth degree. However, due to the orthogonal symmetry it takes more simple form

$$
\operatorname{det}\left(\boldsymbol{q}_{ \pm} \boldsymbol{q}_{ \pm}^{\dagger}-\underline{\rho}\right)=\left(\underline{\rho}^{2}-K_{0} \underline{\rho}+K_{1}\right)^{2}
$$

and reduces to a polynomial of second order. Now the coefficients $K_{j}$ are given by

$$
\begin{aligned}
& K_{0}=\operatorname{tr}\left(\boldsymbol{q}_{ \pm} \boldsymbol{q}_{ \pm}^{\dagger}\right)=\sum_{1 \leq i<j \leq 4}\left|q_{i j ; \pm}\right|^{2} \\
& K_{1}=\left(\operatorname{det}\left(\boldsymbol{q}_{ \pm} \boldsymbol{q}_{ \pm}^{\dagger}\right)\right)^{1 / 2}=\left|q_{13}^{ \pm} q_{24}^{ \pm}-q_{34}^{ \pm} q_{12}^{ \pm}-q_{23}^{ \pm} q_{14}^{ \pm}\right|^{2} .
\end{aligned}
$$


Again it is obvious that both coefficients $K_{0}>0$ and $K_{1}>0$ are positive and therefore both roots of the characteristic polynomial are positive. So we again have the same two options listed above. The difference between the two symmetric spaces on the level of spectra of $L_{ \pm}$consists in the fact that in the $\mathfrak{s o}(8)$ case each of the roots has multiplicity 2 . Therefore, the multiplicities of the continuous spectra of $\mathfrak{s o}(8)$ MNLSE are twice higher than for the $\mathfrak{s o}(4)$ case.

\subsection{The Principal Minors and Fundamental Representations}

The function $D^{+}(\lambda)$ is analytic function of $\lambda$ in $\Re^{+}$which generates the integrals of motion for the MNLSE. Using the properties of the fundamental representations of the $C_{r}$ and $D_{r}$ series we have [10]

$$
\left\langle\omega_{j}|T(\lambda)| \omega_{j}\right\rangle=\left\langle\omega_{j}\left|D^{+}(\lambda)\right| \omega_{j}\right\rangle=\exp \left(\left(\omega_{j}, \vec{\delta}^{+}(\lambda)\right)\right)
$$

where $\omega_{j}$ is the $j$-th fundamental weight of $\mathfrak{g}$ and $\vec{\delta}^{+}(\lambda)=\sum_{k=1}^{r} \delta_{k}^{+}(\lambda) e_{k}$. Note that the simple roots $\alpha_{k}$ and the fundamental weights $\omega_{j}$ satisfy the relation

$$
2\left(\omega_{j}, \alpha_{k}\right) /\left(\alpha_{k}, \alpha_{k}\right)=\delta_{j k}
$$

More specifically for our examples we have

$$
\delta_{1}^{+}(\lambda)=\ln T_{11}(\lambda), \quad \delta_{2}^{+}(\lambda)=\ln \left(\begin{array}{ll}
1 & 2 \\
1 & 2
\end{array}\right)_{T(\lambda)}
$$

for $\mathfrak{s p}(4)$ and

$$
\begin{aligned}
\delta_{1}^{+}(\lambda)=\ln T_{11}(\lambda), & \delta_{2}^{+}(\lambda)=\ln \left\{\begin{array}{ll}
1 & 2 \\
1 & 2
\end{array}\right\}_{T(\lambda)} \\
\delta_{3}^{+}(\lambda)=\ln \left\{\begin{array}{lll}
1 & 2 & 3 \\
1 & 2 & 3
\end{array}\right\}_{T(\lambda)}-\delta_{4}^{+}(\lambda), & \delta_{4}^{+}(\lambda)=\frac{1}{2} \ln \left\{\begin{array}{llll}
1 & 2 & 3 & 4 \\
1 & 2 & 3 & 4
\end{array}\right\}_{T(\lambda)}
\end{aligned}
$$

for $\mathfrak{s o}(8)$. Here by $\left\{\begin{array}{lll}1 & \ldots & k \\ 1 & \ldots & k\end{array}\right\}_{T(\lambda)}$ we denote the upper principal minor of order $k$ of the scattering matrix $T(\lambda)$.

Note that due to the orthogonal symmetry inherent in $D_{4}$ all functions $\exp \left(\delta_{k}^{+}(\lambda)\right)$ are polynomial expressions in terms of the matrix elements of $T(\lambda)$. 


\section{Discussion}

Here we briefly outline another important application of the FAS which is based on the Wronskian relations. It allows one to analyze the mapping $\mathcal{F}: \mathcal{M} \rightarrow \mathcal{T}$ between the class of allowed potentials $\mathcal{M}$ and the scattering data $\mathcal{T}$ of $L$ [4,5]. They are the tool that allows us to show that the Inverse Scattering Method (ISM) is a generalized Fourier transform. For the constant boundary conditions case they require an additional regularization. Therefore we use the identity $[3,13]$

$$
\left.\widehat{\chi}(q(x, t)-\lambda J) \chi(x, \lambda)\right|_{x=-\infty} ^{\infty}=-\mathrm{i} \int_{-\infty}^{\infty} \mathrm{d} y \widehat{\chi}(y, \lambda) q_{y} \chi(y, \lambda)
$$

which follows from equation (2). Here $\chi(x, \lambda)$ can be any fundamental solution of $L$. For convenience we choose it to be the FAS in the region $\mathcal{R}_{1}$. The left hand side of (153) can be calculated explicitly by using the asymptotic of FAS for $x \rightarrow \pm \infty$. It would be expressed by the matrix elements of the factors $S^{+}(\lambda)$, $T^{-}(\lambda)$ and $D^{+}(\lambda)$ which determine the scattering data of $L$. Thus for our first example

$$
\begin{aligned}
& s_{12 ; p}^{+}(z)=-\frac{\mathrm{i} z}{\rho} \int_{-\infty}^{\infty} \mathrm{d} y \operatorname{tr}\left(q_{y} \chi E_{p+1,1} \hat{\chi}(x, z)\right) \\
& s_{23 ; p}^{+}(z)=\frac{\mathrm{i}}{\rho z} \int_{-\infty}^{\infty} \mathrm{d} y \operatorname{tr}\left(q_{y} \chi E_{n+1, p+1} \hat{\chi}(x, z)\right)
\end{aligned}
$$

where $E_{k m}$ is an $(n+1) \times(n+1)$ matrix given by $\left(E_{k m}\right)_{p l}=\delta_{k p} \delta_{m l}$. Note that the integrands in the right hand sides of equations (154) are analytic functions in $z$ in the region $\mathcal{R}_{1}$ that tend to zero for $y \rightarrow \pm \infty$. This ensures the existence of the integrals.

The second set of Wronskian relations which we consider relates the variation of the potential $\delta q$ to the corresponding variations of the scattering data $\delta \rho$ and $\delta \mathcal{T}$. For this purpose we use the identity

$$
\left.\widehat{\chi} \delta \chi(x, \lambda)\right|_{x=-\infty} ^{\infty}=-\int_{-\infty}^{\infty} \mathrm{d} y \widehat{\chi} \delta q(y) \chi(y, \lambda) .
$$

Here we consider the special class of variations of $q(x)$ which preserve $\boldsymbol{q}_{ \pm}$, i.e., 
$\delta \boldsymbol{q}_{ \pm}=0$. Then $\delta w_{0, \pm}=0$ and we arrive at

$$
\begin{aligned}
\delta s_{12 ; p}^{+}(z) & =\mathrm{i} \int_{-\infty}^{\infty} \mathrm{d} y \operatorname{tr}\left(\delta q(y) \chi E_{p+1,1} \hat{\chi}(x, z)\right) \\
\delta s_{23 ; p}^{+}(z) & =\mathrm{i} \int_{-\infty}^{\infty} \mathrm{d} y \operatorname{tr}\left(\delta q(y) \chi E_{n+1, p+1} \hat{\chi}(x, z)\right) \\
\delta \ln m_{1}^{+}(z) & =\mathrm{i} \int_{-\infty}^{\infty} \mathrm{d} y \operatorname{tr}\left(\delta q(y) \chi E_{1,1} \hat{\chi}(x, z)\right) .
\end{aligned}
$$

We finish by some comments on the Hamiltonian properties of equation (1). We already mentioned the nontrivial structure of the space of allowed potentials $\mathcal{M}$ which plays the role of a phase space for the MNLSE, due to the nontrivial boundary conditions. It reflects also on the integrals of motion and on the Hamiltonian vector fields that they generate. To be more specific we list the first three integrals of motion of equation (1)

$$
\begin{aligned}
& I_{1}=\frac{1}{2} \int_{-\infty}^{\infty} \mathrm{d} y\left(\left(\boldsymbol{q}^{\dagger}, \boldsymbol{q}(y)\right)-\rho^{2}\right) \\
& I_{2}=\frac{\mathrm{i}}{4} \int_{-\infty}^{\infty} \mathrm{d} y\left(\left(\boldsymbol{q}_{y}^{\dagger}, \boldsymbol{q}(y)\right)-\left(\boldsymbol{q}^{\dagger}, \boldsymbol{q}_{y}\right)\right) \\
& I_{3}=\frac{3}{8} \int_{-\infty}^{\infty} \mathrm{d} y\left(\left(\boldsymbol{q}_{y}^{\dagger}, \boldsymbol{q}_{y}\right)+\left(\boldsymbol{q}^{\dagger}, \boldsymbol{q}(y)\right)^{2}-\rho^{4}\right) .
\end{aligned}
$$

Note that though all the integrals in (157) are convergent, their variational derivatives $\delta I_{k} / \delta \boldsymbol{q}^{\dagger}$ are not necessarily vanishing for $x \rightarrow \pm \infty$. This is true only for $\delta I_{2} / \delta \boldsymbol{q}^{\dagger}$. Therefore, in order to generate regular Hamiltonian dynamics $I_{3}$, which is the candidate for a Hamiltonian for (1), needs to be regularized. This can be done by using one additional integral of motion which is proportional to $I_{1}$ for the scalar case [19]

$$
\tilde{I}_{1}=\int_{-\infty}^{\infty} \mathrm{d} y\left(\left(\boldsymbol{q}^{\dagger}, \boldsymbol{q}_{ \pm}\right)\left(\boldsymbol{q}_{ \pm}^{\dagger}, \boldsymbol{q}(y)\right)-\rho^{4}\right) .
$$

Then we obtain the Hamiltonian of (1) to be

$$
\begin{aligned}
& H_{\mathrm{reg}}=\frac{8}{3} I_{3}-\rho^{2} I_{1}-\tilde{I}_{1} \\
& =\int_{-\infty}^{\infty} \mathrm{d} y\left(\left(\boldsymbol{q}_{y}^{\dagger}, \boldsymbol{q}_{y}\right)+\left(\boldsymbol{q}^{\dagger}, \boldsymbol{q}\right)^{2}-\rho^{2}\left(\boldsymbol{q}^{\dagger}, \boldsymbol{q}\right)-\left(\boldsymbol{q}^{\dagger}, \boldsymbol{q}_{ \pm}\right)\left(\boldsymbol{q}_{ \pm}^{\dagger}, \boldsymbol{q}\right)+\rho^{4}\right) .
\end{aligned}
$$

The regularized Wronskian relations along with the FAS of $L$ can be used to construct the 'squared solutions' and then to interpret the ISM as a generalized Fourier transform. For the scalar case see [24]. 
It will be instructive to derive the dark-dark and dark-bright soliton solutions [26] of (1) by modifying the dressing Zakharov-Shabat method [30] to systems of the form (132), or by using the Darboux transformation method [7].

Another challenge is to extend this analysis also for the class of Lax operators whose potentials take values in Lie super-algebras, see $[6,20]$ and the references therein. This will allow one to treat the supersymmetric generalizations of MNLS and $N$-wave equations with constant boundary conditions.

\section{References}

[1] Ablowitz M., Kaup D., Newell A. and Segur H., The Inverse Scattering Transform - Fourier Analysis for Nonlinear Problems, Stud. Appl. Math. 53 (1974) 249-315.

[2] Ablowitz, M. J., Prinari, B. and Trubatch A., Discrete and Continuous Nonlinear Schrödinger Systems, Cambridge University Press, Cambridge, 2004.

[3] Atanasov V. A. and Gerdjikov V. S., On the Multi-component Nonlinear Schrödinger Equation with Constant Boundary Conditions, In: Gravity, Astrophysics and Strings'05, P. P. Fiziev and M. D. Todorov (Eds), St Kliment Ohridsky University Press, Sofia, 2006, pp. 22-36.

[4] Calogero F. and Degasperis A., Nonlinear Evolution Equations Solvable by the Inverse Spectral Transform I, Nuovo Cim. B 32 (1976) 201-242.

[5] Calogero F. and Degasperis A., Nonlinear Evolution Equations Solvable by the Inverse Spectral Transform II, Nuovo Cim. B 39 (1976) 1-54.

[6] Chaichian M. and Kulish P. On the Method of Inverse Scattering Problem and Backlund Transformations for Supersymmetric Equations, Phys. Lett. B 78(1978) 413-416.

[7] Degasperis A. and Lombardo S., Multicomponent Integrable wave Equations: I. Darboux-dressing Transformation, J. Phys. A: Math. \& Theor. 40 (2007) 961-977.

[8] Fordy A. and Kulish P., Nonlinear Schrödinger Equations and Simple Lie Algebras, Commun. Math. Phys. 89 (1983) 427-443;

Athorne, C. and Fordy A., Generalized KdV and MKdV Equations Associated with Symmetric Spaces, J. Phys. A: Math. \& Gen. 20 (1987) 1377-1386.

[9] Gerdjikov V. S., On the Spectral Theory of the Integro-Differential Operator $\Lambda$, Generating Nonlinear Evolution Equations, Lett. Math. Phys. 6 (1982) 315-324. 
[10] Gerdjikov V. S., Generalised Fourier Transforms for the Soliton Equations. Gauge Covariant Formulation, Inverse Problems 2 (1986) 51-74.

[11] Gerdjikov V. S., Algebraic and Analytic Aspects of N-Wave Type Equations, Contemporary Mathematics 301 (2002) 35-68.

[12] Gerdjikov V. S., Basic Aspects of Soliton Theory, In: Geometry, Integrability and Quantization, I. M. Mladenov and A. C. Hirshfeld (Eds), Softex, Sofia, 2005, pp. 78-125 (ArXiv:nlin.SI/0604004).

[13] Gerdjikov V. S., Selected Aspects of Soliton Theory. Constant Boundary Conditions, In: Prof. G. Manev's Legacy in Contemporary Aspects of Astronomy, Gravitational and Theoretical Physics, V. Gerdjikov and M. Tsvetkov (Eds), Heron Press Ltd, Sofia, 2005, pp. 277-290 (n lin. S I / $0604004)$.

[14] Gerdjikov V. S., Grahovski G. G. and Kostov N. A., Reductions of N-Wave Interactions Related to Low-Rank Simple Lie Algebras. I: $\mathbb{Z}_{2}$-reductions, J. Phys. A: Math. \& Gen. 34 (2001) 9425-9461.

[15] Gerdjikov V. S. and Khristov E. Kh., On the Evolution Equations Solvable with the Inverse Scattering Problem. I. The Spectral Theory, Bulg. J. Phys. 7 (1980) 28-41;

- On the Evolution Equations Solvable with the Inverse Scattering Problem. II. Hamiltonian Structures and Bäcklund Transformations, Bulg. J. Phys. 7 (1980) 119-133.

[16] Gerdjikov V. S. and Kostov N. A., Multicomponent MKdV Equations on Symmetric Spaces of DIII-type and Their Reductions, In: Geometry, Integrability and Quantization, I. M. Mladenov and M. de Leon (Eds), Softex, Sofia, 2008, pp.36-65.

[17] Gerdjikov V. S., Kostov N. A. and Valchev T. I., On Multicomponent NLS Equations with Constant Boundary Conditions, Theor. Math. Phys. (2009) (In press).

[18] Gerdjikov V. S. and Kulish P. P., Completely Integrable Hamiltonian Systems Related to the Non-Self-Adjoint Dirac Operator (In Russian), Bulgarian J. Phys. 5 (1978) 337-349;

- A Method for the Derivation of the Bäcklund Transformation in the Formalism of the Inverse Scattering Method (In Russian), Theor. Math. Phys. 39 (1979) 69-74.

[19] Gerdjikov V. S. and Kulish P. P., On the Multicimponent Nonlinear Schrödinger Equation in the Case of Non-Vanishing Boundary Conditions, 
Sci. Notes of LOMI Seminars 131 (1983) 34-46; English translation: J. Sov. Math. 30 (1985) 2261-2269.

[20] Gürses M. , Oguz Ö. and Salihoglu S., Nonlinear Partial Differential Equations on Homogeneous Spaces, International Journal of Modern Physics A, 5 (1990) 1801-1817.

[21] Helgasson S., Differential Geometry, Lie Groups and Symmetric Spaces. Graduate Studies in Mathematics vol. 34, AMS, Providence, Rhode Island, 2001.

[22] Ieda J., Miyakawa T. and Wadati M., Exact Analysis of Soliton Dynamics in Spinor Bose-Einstein Condensates, Phys. Rev. Lett. 93 (2004) 194102.

[23] Kaup D. J. and Newell A. C., Soliton Equations, Singular Dispersion Relations and Moving Eigenvalues, Adv. Math. 31 (1979) 67-100.

[24] Konotop V. V. and Vekslerchik V. E., Direct Perturbation Theory for Dark Solitons, Phys. Rev. E 49 (1994) 2397-2407.

[25] Manakov S. V., On the Theory of Two-Dimentional Stationary Self-Focusing of Electromagnetic Waves, Sov. Phys. JETF 38 (1974) 248-253.

[26] Prinari B., Ablowitz M. and Biondini G., Inverse Scattering Transform for the Vector Nonlinear Schrödinger Equation with Nonvanishing Boundary Conditions, J. Math. Phys. 47 (2006) 063508.

[27] Shabat A. B. The Inverse Scattering Problem for a System of Differential Equations (In Russian), Functional Annal. \& Appl. 9 (1975) 75-78;

- The Inverse Scattering Problem (In Russian), Diff. Equations 15 (1979) 1824-1834.

[28] Takhtadjan L. A. and Faddeev L. D., Hamiltonian Approach to Soliton Theory, Springer, Berlin, 1986.

[29] Zakharov V. E. and Manakov S. V., On the Complete Integrability of the Nonlinear Schrödinger Equation (In Russian), Theor. Math. Phys. 19 (1974) 332-343.

[30] Zakharov V. E., Manakov S. V., Novikov S. P. and Pitaevskii L. I., The Theory of Solitons. The Inverse Transform Method (In Russian), Nauka, Moscow, 1980.

[31] Zakharov V. E. and Shabat A. B., On the Interaction of Solitons in Stable Medium (In Russian), Sov. Phys. JETF 37 (1973) 823-828.

[32] Zakharov V. E. and Shabat A. B., A Scheme for Integrating Nonlinear Equations of Mathematical Physics by the Method of the Inverse Scattering Transform. I, Funct. Ann. \& Appls. 8 (1974) 43-53; 
- A Scheme for Integrating Nonlinear Equations of Mathematical Physics by the Method of the Inverse Scattering Transform. II, Funct. Ann. \& Appls. 13 (1979) 13-23.

Vladimir S. Gerdjikov

Institute of Nuclear Research and Nuclear Energy

Bulgarian Academy of Sciences

Blvd. Tsarigradsko chaussée 72

1784 Sofia, BULGARIA

E-mail address: gerjikoveinrne.bas.bg 